\title{
STOCHASTIC ION HEATING BY LOWER HYBRID TURBULENCE*
}

\author{
J. Candy+ \\ Exploratory Sudies Group \\ Accelerator \& Fusion Research Division \\ Lawrence Berkeley Laboratory \\ 1 Cyclotron Road \\ Berkeley, CA 94720
}

May 1990

\section{DISCLAIMER}

\begin{abstract}
This report was prepared as an account of work sponsored by an agency of the United States Government. Neither the United States Government noi an" agency thereof, nor any of their employees, makes any warranty, express or implied, of assuises any legal liability or responsibility for the accuracy, completeness, or usefulness of any information, apparatus, producl, or prosess disclosed, or represents tha! its use would not infringe privately owned rights. Refer. ence herein to any specific commercial product, process, or service by trade name, trademark. manufacturer, or otherwise does not necessarily constitute or imply its endorsement, recommendation, or favoring by the United States Government or any agency therecf. The views and opinions of authors expressed herein do not necessarily state or reflect those of the United Stutes Gowernment or any agency thereof.
\end{abstract}

This work was supported by the Natural Sciences and Engineering Research Council of Canada, and also by the Director, Office of Energy Rescarch, Office of Basic Enerigy Sciences, Materials Sciences Division, of the U S. Department of Energy under Contract No. DE-AC03-76SF00098.

+ Permanent Address: Dept. of Physics, University of Alberta, Edmonton, Alberta, Canada T6G 2G1 


\title{
STOCHASTIC ION HEATING BY LOWER HYBRID TURBULENCE
}

\author{
J. Candy
}

\begin{abstract}
The motion of an ion in a spectrum of lower hybrid waves propagating across a constant magnetic field is examined. In particular, numerical simulation is used to determine the extent to which a turbulent spectrum of these clectrostatic waves may accelerate thermal ions $(T<1 \mathrm{eV})$. The significance of stochastic web development in this hearing process is also discussed.
\end{abstract}




\section{Introduction}

Consider a particle with mass $m$ and charge e gyrating in a uniform magnetic field $B \hat{\mathbf{z}}$ and interacting with an electrostatic wave. The magnetic field in this case may be derived from the vector potential $A=B x y$. Then, if the wave has amplitude $E$ and spatial frequency $\omega$, the Hamiltonian of the particle can be written as

$$
H=\frac{1}{2 m}\left[(m \Omega x)^{2}+p_{x}^{2}+p_{x}^{2}\right]+\frac{e E}{k} \cos \left(k_{x} x+k_{z} z-\omega t\right),
$$

where $\Omega \equiv e B / m$ is the cyclotron frequency. Note that we have chosen the wavevector $\mathbf{k}=k_{x} \hat{x}+k_{z} \hat{z}$ to lie in the $x-z$ plane. That this results in no loss of generality should be clear.

The Hamiltoniar (1) has been studied in some detail by Smith and Kaufman [1], [2]. By considering the overlap of adjacent resonances, these authors obtained a crude estimate of the onset of global stochasticity according to the well-known Chirikov criterion.

More detailed studies, however, have involved a simplified version of (1). If one considers the wave to propagate transverscly $\left(k_{z}=0\right)$, then the provious Hamiltonian function reduces to

$$
H=\frac{1}{2 m}\left[(m \Omega x)^{2}+p_{x}^{2}\right]+\frac{e E}{k} \cos (k x-\omega t) .
$$

This Hamiltonian has been examined in the context of particle heating by a lower hybrid wave - the propagation of which is characterized by $k_{x} \gg k_{z}$ and $\omega \gg \Omega$. Fukuyama et al. $\{3]$ have analysed (2) in the case where $\omega$ is an integer multiple of $\Omega$, while Karney [4] has considered the more general case of arbitrary wave frequency. Futhermore, Karncy has also extended sone of the interesting results obtained for (2) to the uore general Hamiltonian (1). Malkov [5] and Zaslavsky et al. [6], finally, 
have studied the equations of motion which result from (2) in the presence of an arbitrarily weak magnetic field.

More recently, mucl attention has been given to the interesting dynamics which occur when $\omega / \Omega \in \mathbb{Z}$. In this resonant case, the phase space of the system is covered by a stochastic web. Some interesting properties of the separatrix mesh were shown by Chernikov et al. [7] for one wave, and numerically by Murakmi et al. [8] for up to 20 waves. In addition, Karimabadi and Angelopoulos [9] - using first order perturbation theory - have studied invariant curves in phase space for the interaction of a relativistic particle with an obliquely propagating wave packet of arbitrary polarization. They point out certain limitations and weaknesses associated with the usual nonrelativistic treatment.

Finally, by considering a wave packet composed of an infinite number of modes uniformly spaced in frequency, Zaslavsky et al. [10] were able to construct an exact mapping from the continuous Hamiltonian. The iterates of this mapping generate a web with remarkable symmetry properties and fractal-like structure. In fact, the geometry of the separatrix mesh in phase space is reminiscent of a Penrose tiling (see [11], [12]). An interesting summary of stochastic webs in general may be found in [13]. Unfortunately, the structure of the electric fields which give rise to such interesting dynanics are often difficult to justify from a physical standpoint. 


\section{A Model of Lower Hybrid Turbulence}

Particle heating in the earth's ionosphere is believed to result from the interaction of ions with lower hybrid waves. (see [14] and [15]). In particular, precipitating electron beams in this region of the ionosphere might constitute a natural free energy source for the generation of lower hybrid waves. Unfortunately, the general problem of particle heating is enormously complicated, and it is no surprise that many aspects of these processes are still poorly understood. In what follows, we will investigate a single particle model of heating which is similar in form to, but slightly more general than the system (2). Further, we will examine the behavior of our model using physical parameters which are believed to be characteristic of a plasma containing such lower hybrid waves.

\subsection{The Model Hamiltonian}

To begin with, we consider a spectrum of $N$ transversely propagating electrostatic waves. This case is a simple generalization of (2), with the electric field now described by the wave packet

$$
E(x, t)=\sum_{i=1}^{N} E_{i} \sin \left(k_{i} x-\omega_{i} t+\varphi_{i}\right) .
$$

The Hamiltonian for a particle subject to this more general electric field can be written

$$
\mathcal{H}=\frac{1}{2}\left(x^{2}+p^{2}\right)+\sum_{i=1}^{N} \frac{\epsilon_{i}}{k_{i}} \cos \left(k_{i} x-\nu_{i} T+\varphi_{i}\right) .
$$

Note that we have introduced the dimensioniess time $T \equiv \Omega t$ and the canonical momentum $p \equiv d x / d T=p_{x} / m \Omega$, so that $\mathcal{H}$ has dimensions of (lengtll $)^{2}$. The parameter $\nu_{i}$ is related to the temporal frequency $\omega_{i}$ of the $i^{\text {th }}$ wave by the relationship

$$
\nu_{i} \equiv \frac{\omega_{i}}{\Omega}
$$


Furthermore, for a plasma composed of singly ionized hydrogen, the noniznearity parameter $\epsilon_{i}$ is defined in terms of the mode amplitude $E_{i}$ according to

$$
\epsilon_{i} \equiv \frac{e E_{i}}{m_{p} \Omega^{2}} .
$$

Also, in each wave we have included an arbitrary phase factor $\varphi_{i}$ which can be set to any value in the range $0 \leq \varphi_{i}<2 \pi$. In principle, all that remains is to choose reasonable values for the parameters $\epsilon_{i}, k_{i}, \nu_{i}$ and $\Omega$ so that the equations of motion corresponding to (4) may be solved numerically.

\subsection{Limiting the Parameter Space}

At altitudes between $500 \mathrm{~km}$ and $2500 \mathrm{~km}$ the background magnetic field varies from about $4 \times 10^{-5}$ Tesla to $2 \times 10^{-5}$ Tesla. In general, we will assume that $B$ assumes a constant value of

$$
B=4 \times 10^{-5} \text { Tesla, so that } \Omega=3.8318 \times 10^{3} \mathrm{~s}^{-1} \text {, }
$$

corresponding to a lower altitude field strength. Typical measured electric field amplitudes are on the order of $0.01 \mathrm{~V} / \mathrm{m}$ [16]. Significantly higher amplitudes are indeed possible, and we consider (arguably high) maximum values on the order of $1 \mathrm{~V} / \mathrm{m}$ for $E(x, t)$. This impiies that the normalized maximuın value of the field (see $(5)$ ) is

$$
\frac{e E\left(x_{1} t\right)}{m_{p} \Omega^{2}} \sim 10 \mathrm{~m}
$$

(Actually, we will consider this to be an upper bound on the RMS value of ilic normalized field. This is discussed at length in Section 3.3). Such a restriction provides a rough guideline for choosing the set of nonlinearity parameters $\epsilon_{i}$.

Next, to decide upon a reasonable value for each $\nu_{i}$ we must make use of the 
dispersion relation for $\mathrm{LH}$ waves:

$$
\omega=\omega_{p i}\left(1+\frac{\omega_{p e}^{2}}{\omega_{g e}^{2}}\right)^{-1 / 2}\left(1+\frac{m_{p}}{m_{e}} \sin ^{2} \theta\right)^{1 / 2},
$$

where

$$
\omega_{p i}^{2}=\frac{n_{0} e^{2}}{\varepsilon_{0} m_{p}}, \quad \omega_{p e}^{2}=\frac{n_{0} e^{2}}{\varepsilon_{0} m_{e}}, \quad \omega_{g e}^{2}=\frac{B^{2} e^{2}}{m_{e}},
$$

and $\tan \theta=k_{z} / k_{x}$. These expressions show the explicit dependence of $\omega$ on the local density $n_{0}$.

The dispersion relation (6) is valid for small $\theta$ (i.e., $k_{z} \ll k_{x}$ ). Also, it is true that the Hamiltonian (4) - to a good approximation - describes the ion motion in the $x-y$ plane even for small but nonzero $k_{z}$. Of course, the price of this simplification is to forego any detailed knowledge of the parallel motion. Fortunately, our concern here is with perpendicular acceleration only, so that this loss of information is of little concern. As a result, (4) affords us a valid picture of the perpendicular motion even beyond the $k_{x}=0$ approximation.

Upon dividing (6) by $\Omega$ and squaring the result, one obtains

$$
\left(\frac{\omega}{\Omega}\right)^{2}=m_{p}\left(\frac{\varepsilon_{0} B^{2}}{n_{0}}+m_{e}\right)^{-1}\left(1+\frac{m_{p}}{m_{e}} \sin ^{2} \theta\right) .
$$

In the region of the ionosphere we are interested in, realistic densities range from $10^{8} \mathrm{~m}^{-3}$ to $10^{10} \mathrm{~m}^{-3}$. Then, according to $(7)$, the interval $0 \leq \theta \leq \pi / 60$ corresponds to the following ranges in $\omega / \Omega$ :

$$
\begin{array}{rll}
3.4 \leq \frac{\omega}{\Omega} \leq \mathrm{S} .4 & \text { for } & n_{0}=10^{8} \mathrm{~m}^{-3} \\
26.8 \leq \frac{\omega}{\Omega} \leq 65.8 & \text { for } & n_{0}=10^{10} \mathrm{~m}^{-3}
\end{array}
$$

Using these limits as rough guidelines, we assume that $\nu_{i}$ may lie anywhere in the range

$$
4 \leq \nu_{i} \leq 30
$$


It remains to find a physically reasonable value for each wavenumber $k_{i}$. Unfortunately, no physical measurements of the wavevectors of ionospheric lower hybrid waves have been obtained. It seems the best we can do is to consider possible driving sources of the waves, and derive a theoretical estimate. According to Koskinen [16], values of $k_{i}$ on the order of $1 \mathrm{~m}^{-1}$ may be obtained by assuming that the $\mathrm{LH}$ waves are excited by a parallel beam of $4 \mathrm{keV}$ clectrons. Hence, we assume each $k_{i}$ is restricted to the interval

$$
0.1 \mathrm{~m}^{-1} \leq k_{i} \leq 1.0 \mathrm{~m}^{-1}
$$

\subsection{Initial Conditions of Source Ions}

Using the canonical variables we defined earlier, the ion gyroradius $\rho$, defined by

$$
\rho^{2} \equiv \frac{1}{\Omega^{2}}\left[\left(\frac{d x}{d t}\right)^{2}+\left(\frac{d y}{d t}\right)^{2}\right],
$$

takes on the simple form

$$
\rho=\sqrt{\mathrm{p}^{2}+x^{2}}
$$

To see this, one must realize that in the derivation of (1), $p_{y}$ was chosen to have a constant initial value of zero, thus implying

$$
p_{y}=m \frac{d y}{d t}+m \Omega x=0 .
$$

The perpendicular kinetic encrgy (or temperature) $T$ of a test ion is, of course, related to the square of the gyroradius by

$$
\tau=\frac{m_{p}}{2 e}(\Omega \rho)^{2} \quad[\text { in } \mathrm{eV}] .
$$

Typical ionospheric source ions have hinetic energies on the order of a fraction of an $\mathrm{eV}$ [14]. Let us assume that the ions we are considering have, in their perpendicular degrees of freedom, kinetic encrgies between $0.25 \mathrm{cV}$ and $1 \mathrm{eV}$. This range 
in energy corresponds corresponds to gyroradii in the interval

$$
1.8 \mathrm{~m} \leq \rho \leq 3.6 \mathrm{~m}
$$

\subsection{Method of Integration}

To study the time evolution of $p$ and $x$, we can generate a symplectic integration algorithm using the Hamiltonian function (4). Specifically, ve will use the SIA4 algorithm derived in [17]. This algorithm is accurate to $4^{\text {th }}$ order in the time step, and is one of a class of symplectic integrators devised originally by Ruth [18], [19]. With (4) written in the form $\mathcal{K}=\mathcal{H}_{1}(p)+\mathcal{H}_{2}(x, T)$, the SIA uses the functions

$$
F(x, T) \equiv-\partial_{x} \mathcal{H}_{2}(x, T)=\sum_{i=1}^{N} \epsilon_{i} \sin \left(k_{i} x-\nu_{i} T+\varphi_{i}\right)-x
$$

and

$$
P(p) \equiv \partial_{p} \mathcal{H}_{1}(p)=p
$$

to construct an explicit, analytic symplectic map. This symplectic map approximates the exact phase flow induced by $\mathcal{H}$, accurate to fourth order in the time step. The advantages of using SIA4 in place of a more common non-symplectic integrator are many, and are discussed at length in [17]. Further details concerning the integration procedure will be discussed later. 


\section{Numerical Results}

Using the SIA4 algorithm, we studied the time development of our model system for a variety of parameter values. A discussion of the results of this study appears below, with a separate section devoted to each particular simulation. Furthermore, a collection of figures which summarize the respective simulations appears at the end of this paper, supplemented by captions which describe in full the associated parameter spaces.

The time steps $\delta T$ used in SIA4 had the form

$$
\delta T=\frac{\Delta T}{j}, \text { where } \Delta T \equiv \frac{2 \pi}{\bar{\nu}} .
$$

In the above,

$$
\bar{n} \equiv\left\{\text { the integer nearest to } \max \left(\nu_{1}, \ldots, \nu_{N}\right)\right\},
$$

and $j$ is an arbitzary integer characterizing the smallness of the time step - and hence the accuracy of the sympiectic integrator. Generally, $j$ was chosen to lie between about 20 and 25; a size at which, loosely speaking, it is probably valid to forget about the original Hamiltonian and consider the system as described exac:ly by the symplectic map SIA4.

\subsection{Heating with One Mode}

\subsubsection{Surfaces of Section}

We begin by studying the time evolution of is system corresponding to the Hamiltonian (4) for the simplest cass: of $N=1$. Figures 1 and 2 illustrate tire structure of phase space for this case, with all parameters equal except for $\nu_{1}$. In particular, the first figure has $\nu_{1}=5$, while the second has $\nu_{1}=5.16$. In both cases, the wave 
amplitudes are relatively small, leaving much of the stase space covered by invariant tori. However, the two cases have clearly distincti topologies. In the first case, there exists a narrow chanuel of interconnected separatrices which form a web that spans the entire plase plane. This is in contrast to the picture in Figure 2, which displays a series of nested tori as predicted by the KAM theorem. Interestingly, the violation of the KAM theorem in the first case, as a consequence of $\nu_{1}$ being an integer, allows a theoretical diffusion along ihe stochastic network to arbitrarily large energies. However, the thickness of the web decreases rapidly with increasing velocity, so that diffusion is quite limited in practice.

Next, Figures 3 and 4 illustrate the effect of increasing the strength of the electrostatic wave. By comparing Figure 3 to Figure 1, it beromes clear that the increase in wave amplitude produces a widening of the stochastic network, while in Figure 4, one can see that it leads to a breakup of invariant tori. Finally, a characteristic common to all of these first four figures is the existence of a sequence of little tori enclosing the origin which effectively trap low energy particles. We will comment on the significance of such barriers in inhibiting particle acceleration later.

\subsubsection{Er: :emble Averaging}

Next, we wish to examine the average phase space population $N(\rho)$ which results from accelerating an ensemble of low energy particles using the Hamiltonian (4). The steps taken to determine this quantity are described below:

- Assign 10 ions random positjons in the phnse sjonce annulus 1.8 in $\leq \rho \leq 3.6 \mathrm{~m}$ at $T=0$.

- Integrate cach particle in this ensemble forwaral in time to $T=200 \pi$. 
- From $T=200 \pi$ to $T=2000 \pi$, record the position of each particle at successive time intervals $\Delta T=2 \pi / \bar{\nu}($ see $(9))$.

Figures 4 through 9 illustrate this averaging procedure for fixed values of $\nu_{1}$, $k_{1}$ and $\varphi_{1}$, but with successively increasing wave amplitude $\epsilon_{1}$. In the figures, $N(\rho)$ is expressed in arbitrary units. The local depletion of particles in each case corresponds to the presence of large first-order islands such as those in Figure 1. In the limit of sinall wave amplitude, the elliptic fised points exist at gyroradii $\rho$ which satisfy

$$
J_{n}^{\prime}\left(k_{1} \rho\right)=0 .
$$

As the wave amplitude grows to its maximum value, successive elliptic points are encircled, while the distributions around previous fixed points become smeared as invariant curves are destroyed.

The same simulation, with $\nu_{1}=5.16$, exhibits essentially no growth in energy. This can be seen in Figures 10 and 11, and results from the entire ensemble remaining trapped by invariant tori.

However, the situation changes if we double the wavevector $k_{1}$ to unity. Figure 12 $\left(\nu_{1}=5\right)$ and Figure $13\left(\nu_{1}=5.16\right)$ illustrate the results of a simulation which considered only one ion averaged over the period $T=200 \pi$ to $T=6000 \pi$, with $\Delta T$ defined as before. These results show conparable heating profiles for both values of $\nu_{1}$. The increased magnitude of $k_{1}$ effectively reduces the phase velocity of each wave $n d$, in the case of noninteger $\nu_{1}$, destroys tori closer to the origin and allows stochastic diffusion of the test ion. 


\subsection{Amplitudes of Ordered and Turbulent Wave Spectra}

We claimed in Section 2.2 that the normalized maximum value of the electric field should not exceed roughly $10 \mathrm{~m}$. We did not, however, put particular restrictions on the normalized mode amplitudes $\epsilon_{i}$. Clearly, the maximum possible amplitude of a wave packet composed of $N$ sinusoidal modes, each with anplitude $\epsilon_{i}$, is simply

$$
\sum_{i=1}^{N}\left|\epsilon_{i}\right|
$$

However, in reality, one would expect that such an amplitude would rarely be attained - especially in the case where $\mathrm{N}$ is large.

Consider, first, the normalized electric field seen by the particle at time $T$ :

$$
\mathcal{E}(x(T), T) \equiv \frac{e E(x(T), T)}{m_{p} \Omega^{2}}=\sum_{i=1}^{N} \epsilon_{i} \sin \left(k_{i} x(T)-\nu_{i} T+\varphi_{i}\right)
$$

The RMS value of this field, taken over time interval $[0, \tau]$, is defined as

$$
\mathcal{E}_{\mathrm{rmo}}(\tau) \equiv\left[\frac{1}{\tau} \int_{0} \mathcal{E}^{2}(x(T), T) d T\right]^{1 / 2}
$$

In the limit $k_{i} \rightarrow 0$, and $\tau \rightarrow \infty$, it is simple to calculate $\mathcal{E}_{\mathrm{rm}}$ analytically. Calling this limiting value of the RMS field $\tilde{\varepsilon}_{\text {rmo }}$, it is easy to show that

$$
\bar{\varepsilon}_{r m e}=\left[\frac{1}{2} \sum_{i=1}^{N} \epsilon_{i}^{2}\right]^{1 / 2} \text {. }
$$

The validity of this result also requires that all $\nu_{i}$ are distinct.

We numerically computed $\mathcal{E}_{r m s}\left(10 \pi / \nu_{\min }\right)$ for two different wave packets, each with $N=10$, and compared the results with the approximate value $\vec{\varepsilon}_{\mathrm{rm} .}$. The first case, shown in Figure 14, consists of waves evenly spaced in frequency with constant wavenumber and amplitude. The reader should recognize this spectrum as one which will give rise to a stochastic web. It happens that $\mathcal{E}_{\mathrm{rms}}$ for this ordered packet is $2.2 \mathrm{~m}-$ in close agreement with $\dot{\mathcal{E}}_{\mathrm{rm}}=\sqrt{5} \mathrm{~m} \simeq 2.236 \mathrm{~m}$. It is interesting 
to note that as $N \rightarrow \infty$ in such a packet, the electric ficld seen by the particle approaches a periodic comb of delta functions, and is then described by the mapping of Zaslavsky, et al. [10]. In fact, such periodic impulses, or "kicks", seem to be a generic aspect of chaos in Hamiltonian systems. Potential fields of this type give rise to many popular 2-D symplectic maps of the plane which are known to be chaotic - the Standard and Fermi Maps, for example. Furthermore, various approximate analyses of (2) ([5], for example) have concentrated on constructing a perturbed twist map of the plane which replaces the exact motion by: (i) a kick at $\dot{x}=\omega / k$, and (ii) unperturbed linear oscillation everywhere else, as shown in Figure 31. In a qualitative sense, it can be claimed that if the kick is strong enough, the particle will undergo a substantial phase change which will lead to stochastic inotion. For this reason, it seems that the study of finite and infinite mode stochastic webs aray be useful from the point of view of understanding the generic aspects of particle heating in the ionosphere.

In any case, we also calculated $\mathcal{E}_{\text {rmo }}\left(10 \pi / \nu_{\text {min }}\right)$ for the turbulent spectrum shown in Figure 15. While the structure of the field is much more erratic in this case than in the last, the value $\mathcal{E}_{\mathrm{rms}}=2.3 \mathrm{~m}$ is quite comparable.

These calculations provides us with a rough estimate of the relationship between (10) and $\mathcal{E}_{\mathrm{rm} g}$, and serve to justify the values of $\epsilon_{i}$ used in the multiple mode simulations which follow.

\subsection{Multiple Mode Stochastic Webs}

As we mentioned in the last section, the study of finite mode stochastic webs may contribute to a. better understanding of certain general properties of ionospheric particle heati.,g. With this in mind, we choose to examine three particular cases, cach for realistic values of the various wave parameters. Before doing so, we remind 
the reader of some fundamental ideas in nonlinear dynamics.

First, it has been shown that when the number of degrees of freedom satisfies $n \geq 3$, a separatrix nei may arise which extends throughout phase space. This net, which was predicted by Arnold, forms a stochastic web along which particles may wander chaotically. The nane given to this motion is Arnold diffusion, and rciults from the intersection of invariant torj which do not divide phase space (since $n \geq 3$ ). However, the Hamiltonian (4) represents only $n=3 / 2$ degrees of freedom, thus showing (see Figures 16 to 18) that an Arnold-type diffusion may occur even in the case of minimal dimension for nonintegrability.

\subsubsection{Four Modes}

Shown in Figure 16 is the stochastic web which results from a wave packet composed of four modes. The frequencies are spaced according to $\nu_{i}=5 i$ for $i=1, \ldots, 4$; while the amplitudes and wavevectors are constant. Interestingly, the particle spent most of its time sticking to the tori centered at $\rho \sim 38 \mathrm{~m}$, and consequently the iterates were distributed very nonuniformly in phase space.

\subsubsection{Five Modes}

Next, we consider five modes with equal amplitude, and frequencies which are multiples of 5 - but not spaced as in the last example. In this case, we vary the wavenumber so that waves with equal values of $\nu_{i}$ will have different phase velocities. The effect of this modificntion is shown in Figure 17. We see quite dramatic heating effects, with gyroradii approinching $110 \mathrm{~m}$ (or energies approaching 0.93 $\mathrm{keV}$ ). This is achieved, however, with relatively low amplitude RMS fields (less than $0.5 \mathrm{~V} / \mathrm{m}$ ), and in the absence of what is normally considered to be global stochasticity (i.e., destruction of nearly all invariant tori). 


\subsubsection{Six Modes}

As a final example of stochastic web heating, we add a sixth mode to the previous system. This has the effect, surprisingly, of limiting the maximum energy gain to only about $0.62 \mathrm{keV}$ (see Figure 18). Unfortunately, the tendency of the iterates on the surface of section to explore some areas of the separatrix mesh and not others is marked by a sensitive dependance on both initial conditions and the maximum integration time $T_{\max }$. In instances of very large $T_{\max }$, the areas of the web which are explored also depend on the time step used in SIA4 and on the precision of all floating point operations.

\subsection{Heating by a Completely Turbulent Spectrum}

This firal series of simulations is aimed at modeling particle heating in a "realistic" spectrum of waves. We consider $N=10$ waves, each with normalized frequency $\nu_{i}$, wavenumber $k_{i}$ and phase $\varphi_{i}$ chosen at random inside the intervals described in Section 2.2. Then, we calculate the average population $N(\rho)$ according to the prescription in Section 3.1.2 for various cases claracterized by different values of the $\epsilon_{i}$. We performed 12 separate runs, which had the collective structure shown in Table 1. For the 6 runs labeled by " $A$ ", we chose the wave paranneters

$$
\left\{\nu_{i}^{(A)}, k_{i}^{(\lambda)}, \varphi_{i}^{(A)}\right\}_{i=1}^{10}
$$

which remained constant for all values of the mode amplitudes. Then, for cach A Iun, we chose a set of mode amplitudes (composed of $10 \epsilon_{i}$ ) at randon in the interval $\left[\epsilon_{\min }, \epsilon_{\max }\right]$. For the runs labeled " $\mathrm{B}$ ", we generated new values for the wave parameters and the initial ion distribution, then performed the simulations with the same mode ampritudes as in the $A$ runs. The maximum gyroradius $\rho_{\text {max }}$ 
and corresponding temperature $T_{\max }$ to which the ions were accelerated also appear in Table 1. The distributions $N(\rho)$ appear in Figures 19 to 30.

Each of these simulations was performed on a CRAY X-MP supercomputer, using single precision real variables (14 digit accuracy). The integrator time step was chosen with $j=25$ (see (10)).

Perhaps the first difference which one notices in the heating profiles is the value of $\rho_{\text {max }}$ in runs IB, 2B and $3 B_{;}$it is significantly lower than in the corresponding A runs. This can he explained, however, in terms of the minimum modal phase velocities. The slowest modes in the $A$ and $B$ runs respectively had normalized phase velocities of

$$
\left\{\frac{\nu_{i}^{(A)}}{h_{i}^{(1+1)}}\right\}_{\min } \simeq 5.5 \pi \mathrm{m} \quad \text { and } \quad\left\{\frac{\nu_{i}^{(B)}}{h_{i}^{(B)}}\right\}_{\min } \simeq 7.56 \mathrm{~m} \text {. }
$$

Now, for a particle to be effectively accelerated by a wave, it must be moving with a rclocity not substantially less than the phase velocity of that wave. Otherwise, as seen in Figure 31, no resonant interaction will occur. This effect is manifested in our sinulation, where the faster moving $B$ wave is less effective at providing the first jolts of accelcration: than the slower $A$ wave. This is one aspect of particle acceleration, however. that is greatly enlanced by the presence of a stochastic wob. Because of the large first-order islands creat ed by resonauce, particles which are initially located cluse to the urigin in phase space $(T<1 \mathrm{eV})$ may be strept out to substantially higher energies than would be possible otherwise. This is seen clearly in Figme 1. where the scparatijx mesh which surrounds the first-order islands reaches very close to the rorigin. This effect is not observed in Figure 2. where the inmriant torr divirle: the plinse space into successive annulus-like regions.

Next, it happens that the maximum velocity to which a particle may be accelerated is idso dependent upon the values of the modial pliase velocitics. Tu see 
this, note that in the $A$ and $B$ runs respectively, the maximum normalized phase velocities were

$$
\left\{\frac{\nu_{i}^{(A)}}{k_{i}^{(A)}}\right\}_{\max } \simeq 92.2 \mathrm{~m} \text { and }\left\{\frac{\nu_{i}^{(B)}}{k_{i}^{(B)}}\right\}_{\max } \simeq 67.2 \mathrm{~m} .
$$

The larger phase velocity in case A allows stochastic diffusion to occur at correspondingly larger gyroradii. As a result, for the maximum electric field amplitudes in runs $6 \mathrm{~A}$ and $6 \mathrm{~B}$, case $\mathrm{A}$ exhibits superior particle heating characteristics.

\subsection{The Disappearance of Landau Damping}

Recall that one may define the dielectric response function $\varepsilon(k, \omega)$ of a plasma in terms of the dielectric tensor $\epsilon(k, \omega)$ according to

$$
\varepsilon(k, \omega) \equiv \frac{k \cdot \epsilon(k, \omega) \cdot k}{k^{2}}
$$

For a plasma in a uniform, external magnetic field such as that pertaining to (1), we can write this response as a sum over all ion species $\sigma$ according to [20]

$$
\varepsilon(k, \omega)=1-\sum_{\sigma} \sum_{n=-\infty}^{\infty} \int d^{3} v \frac{F_{\sigma, n}(v)}{n \Omega_{a}+k_{z} v_{z}-\omega-i \eta} .
$$

The explicit form of $F_{\sigma, n}(v)$, which is not importani for our purposes, is determined by the single-particle velocity distribution function $f_{o}(v)$. When the wave propagates obliquely to the magnetic field, the contributions to the response occur at values of $v_{z}$ where the denominator in the integral vanishes. These resonances, of course, are the sources of lineat Landau damping of the plasina wave. However, if we let the wave propagate trausversely to the magnctic ficld as in (2), then the new set of resonances $\omega=n \Omega_{\text {o }}$ are indspendent of $v$ - and the Landau damping vanishes. This phenomenon has been exanined closely in [5] and [0], where it is shown that the paradoxical disappearance of Landau damping is accompanied by 
the emergence of a particular form of nonlinear damping. This new damping is related to the stochastic instability of particle motion. In particular, stochastic web formation is one example of this phenomenon. 


\section{Conclusion}

If lower hybrid waves are indeed responsible for the bulk heating of ions in the ionosphere, we hope that the simulations described herein might help eiucidate some of details of the wave-particle interactions involved. Indeed, by studying this simple single particle model, we can gain a better understanding of the characteristics and limitations of $\mathrm{LH}$ heating.

Unfertunately, as was mentioned earlier on, the processes which we have tried to model in this paper are still quite poorly understood. Further, lower hybrid heating is sy no means the only possible mechanism for ion acceleration in the suprauroral region. While the upper limit of $\tau_{\max } \sim 1 \mathrm{keV}$ (at RMS fields of roughly $1.2 \mathrm{~V} / \mathrm{m}$ ) is of the same order of magnitude as temperatures of observed hot ions populations, more experimental data is needed before researchers are able to construct a truly reliable picture of these ionospheric processes.

\section{Acknowlegments}

I would like to thank Dr. Etienne Forest for inviting me to the Lawrence Berkeley Laboratory, where most of the computational work for this paper was done. As well, I must alsu thank my supervisor Dr. Wojciech Rozmus, whose unbounded enthusiasm and continual support made this analysis possible. 


\section{References}

[1] G. R. Smith and A. N. Kaufman, Phys. Rev. Lett. 34 (1975) 1613.

[2] G. R. Smith and A. N. Kaufman, Phys. Fluids 21 (1978) 2230.

[3] A. Fukuyama, H. Momota, R. Itatani and T. Takizuka, Phys. Rev. Lett. 38 (1977) 701.

[4] C. F. F. Karney, Phys. Fluids 21 (1978) 1584; 22 (1979) 2188.

[5] M. A. Malkov and G M. Zaslavsky, Phys. Lett. 106A (1984) 257.

[6] G. M. Zaslavsky, M. A. Malkov, R. 2. Sagdeev and V. D. Shapiro, Sov. J. Plasma Phys. 12(7) (1986) 453.

[7] A. A. Chernikov, M. Ya. Natenzon, B. A. Petrovichev, R. Z. Sagdeev and G. M. Zaslavsky, Phys. Lett. 122A (1987) 39.

[8] S. Murakami, T. Sato and A. Hasegawa, Physica D, 32 (198S) 259.

[9] H. Karimabadi and V. Angelopoulos, Phys. Rev. Lett. 62 (1989) 2342.

[10] G. M. Zaslavsky, M. Yu. Zakharov, R. Z. Sagdeev, D. A. Usikov and A. A: Chernikov, Sov. Phys. JETP 64(2) (19S6) 204.

[11] N. N. Filonenko, R. Z. Sagdeev and G. M. Zaslavsky, Nuclear Fusion 7 (196i) 253.

[12] A. J. Lichtenberg and B. P. Wood, Phys. Rev. 39, (10S0) 2153.

(13) A. A. Chernikov, R. Z. Sagdeev, D. A. Usikov, M. Yu. Zakharov and G.M. Zaslavsky, Nature 326 (1089) 550. 
[14] G B. Crew and T. Chang, in: Physics of Space Plasmas (1985-87), eds. T. Chang, J. Belcher, J. R. Jasperse and G. B. Crew, SPI Conf. Proc. and Reprint Series, vol. 6 (Scientific Publishers, Cambridge, MA, 1987) p. 55.

[15] T. Chang, G. B. Crew and J. M. Retterer, Computer Physics Communications $49(1988) 61$.

[16] H. E. Koskinen, J. Geophys. Res, 90 (1985) 8361.

[17] J. Candy and W. Rozmus, accepted for publication in J. Comput. Phys.

[18] R. D. Ruth, IEEE Trans. Nucl. Sci., NS-30, (1983) 2669.

[19] E. Forest and R. D. Ruth, accepted for publicatior. in Physica D.

[20] S. Ichimaru, Basic Principles of Plasma Physics, (Benjamin/Cummings, Reading, Mass. 1973) p. 51-59. 


\section{Figure Captions}

1. Surface of section of the Hamiltonian (4) for $N=1$. The wave parameters are $\epsilon_{1}=0.7 \mathrm{~m}, \nu_{1}=5, k_{1}=1 \mathrm{~m}^{-1}$ and $\varphi_{1}=0$. Points are plotted at the times $T_{p}=2 \pi p / \nu_{1}$. Trajectories corresponding to various initial conditions are plotted, and the integration parameter $j$ is 20 .

2. Same as [1], except $\nu_{1}=5.16$.

3. Surface of section of the Hamiltonian (4) for $N=1$. The wave parameters are $\epsilon_{\mathrm{l}}=1.75 \mathrm{~m}, \nu_{1}=5, k_{1}=1 \mathrm{~m}^{-1}$ and $\varphi_{1}=0$. Points are plotted at the times $T_{p}=2 \pi p / \nu_{1}$. Trajectories corresponding to various initial conditions are plotted, and the integration parameter $j$ is 20 .

4. Same as [3], except $\nu_{1}=5.16$.

5. Time-averaged ion distribution $N(\rho)$ versus gyroradius $\rho$ for the Hamiltonian (4). Ten ions in the field of a single $(N=1)$ wave are averaged over the interval $200 \pi \leq T \leq 2000 \pi$. The wave parameters are $\epsilon_{\mathrm{l}}=1 \mathrm{~m}, \nu_{1}=5$, $k_{1}=0.5 \mathrm{~m}^{-1}$ and $\varphi_{1}=0$, and the integration parameter $j$ is 25 .

6. Same as [5], except $\epsilon_{1}=1.5 \mathrm{~m}$.

7. Same as [5], except $\epsilon_{1}=2 \mathrm{~m}$.

8. Same as [5], except $\epsilon_{\mathrm{t}}=2.5 \mathrm{~m}$.

9. Same as [5], except $\epsilon_{1}=3$ in.

10. Time-averaged ion distribution $N(\rho)$ versus gyroradius $\rho$ for the Humiltonian (4). Ten ions in the field of a single $(N=1)$ wave are averaged over the 
intervad $200 \pi \leq T \leq 2000 \pi$. The wave parameters are $\epsilon_{1}=1 \mathrm{~m}, \nu_{1}=5.16$, $k_{1}=0.5 \mathrm{~m}^{-1}$ and $\varphi_{1}=0$, and the integration parameter $j$ is 25 .

11. Same as [10], except $\epsilon_{1}=3 \mathrm{~m}$.

12. Time-averaged ion distribution $N(\rho)$ versus gyroradius $\rho$ for the Hamiltonian (4). One ion in the field of a single $(N=1)$ wave is averaged over the interval $200 \pi \leq T \leq 6000 \pi$. The wave parameters are $\epsilon_{1}=3 \mathrm{~m}, \nu_{1}=5, k_{1}=1 \mathrm{~m}^{-1}$ and $\varphi_{1}=0$, and the integration parameter $j$ is 20 .

13. Same as [12], except $\nu_{1}=5.16$.

14. The normalized electric field $\mathcal{E}(x(T), T)$ as seen by a particle in the field of 10 waves. The wave parameters are $\epsilon_{i}=1 \mathrm{~m}, v_{i}=3 i, k_{i}=1 \mathrm{~m}^{-1}$ and $\varphi_{i}=0$ for $i=1, \ldots, 10$. The horizontal axis shows the number of spatial periods of the slowest wave, and integration parameter $j$ is 20 .

15. The normalized electric field $\mathcal{E}(x(T), T)$ as seen by a particle in the fiel:' of 10 waves. The wave parameters are $\epsilon_{i}=1 \mathrm{~m}$, with $\nu_{i}, k_{i}$ and $\varphi_{i}$ chosen at random for $i=1, \ldots, 10$. The horizontal axis shows the number of spatial periods of the slowest wave, and integration parameter $j$ is 20 .

16. Surface of section of the Hamiltonian (4) for $N=4$. The wave paraneters are $\epsilon_{i}=1.75 \mathrm{~m}, \nu_{i}=5 i, k_{i}=0.7 \mathrm{~m}^{-1}$ and $\varphi_{i}=0$ for $i=1, \ldots, 4$. Points are plotied at the times $T_{p}=2 \pi p / \nu_{1}$, for $p=1, \ldots, 15000$. The initial gyroradius is $2.5 \mathrm{~m}$, and the integration parameter $j=17.5$ is not an integer in this special casc.

17. Surface of section of the Hamiltonian (4) for $N=5$. Wave parameters are $c_{i}=1.8 \mathrm{~m},\left(\nu_{1}, \ldots, \nu_{5}\right)=(5,10,10,15,15),\left(k_{1}, \ldots, k_{5}\right)=(0.7,0.75,0.55,0.7$, 
$0.35) \mathrm{m}^{-i}$ and $\varphi_{i}=0$ for $i=2, \ldots, 5$. Points are plotted at the times $T_{p}=2 \pi p / \nu_{1}$, for $p=1, \ldots, 15000$. The initial gyroradius is $2.6 \mathrm{~m}$, and the integration parameter is $j=22$.

18. Surface of section of the Hamiltonian (4) for $N=6$. Wave parameters are $c_{i}=1.8 \mathrm{~m},\left(\nu_{1}, \ldots, \nu_{6}\right)=(5,10,10,15,15,20),\left(k_{1}, \ldots, k_{\theta}\right)=(0.7,0.75,0.55$, $0.7,0.35,0.7) \mathrm{m}^{-1}$ and $\varphi_{i}=0$ for $i=1, \ldots, 6$. Points are plotted at the times $T_{p}=2 \pi p / \nu_{1}$, for $p=1, \ldots, 15000$. The initial gyroradius is $2.6 \mathrm{~m}$, and the integration paraneter $j=17.5$ is not an integer in this special case.

19. Time-averaged ion distribution $N(\rho)$ versus gyroradius $\rho$ for the Hamiltonian (4). Ten ions in the field of ten waves are averaged over the interval $200 \pi \leq T \leq 2000 \pi$. The wave parameters are chcsen at random according to the prescriptions in the text (case $A$ ), with the mode amplitude range $\left[\epsilon_{\min }, \epsilon_{\text {max }}\right]=[0,1] \mathrm{m}$. The integration parameter $j$ is 25 .

20. Same as [19], except $\left[\epsilon_{\min }, \epsilon_{\max }\right]=[0.5,1.5] \mathrm{m}$.

21. Same as $[19]$, except $\left[\epsilon_{\min }, \epsilon_{\text {max }}\right]=[1,2] \mathrm{m}$.

22. Same as $[19]$, except $\left[\epsilon_{\min }, \epsilon_{\max }\right]=[1.5,2.5] \mathrm{m}$.

23. Same as $[19]$, except $\left[\epsilon_{\min }, \epsilon_{\max }\right]=[2,2.5] \mathrm{m}$.

24. Same as $[19]$, except $\left[\epsilon_{\min }, \epsilon_{\max }\right]=[3,3.5] \mathrm{m}$.

25. Time-averaged jon distribution $N(\rho)$ versus gyroradius $\rho$ for the Hamiltonian (4). Ten ions in the field of ien waves are averaged over the interval $200 \pi \leq T \leq 2000 \pi$. The wave parameters are chosen at random according to the prescriptions in the text (case $\mathrm{B}$ ), with the mode amplitude range $\left[\epsilon_{\min }, \epsilon_{\max }\right]=[0,1] \mathrm{m}$. The integration parameter $j$ is 25 . 
26. Same as [25], except $\left[\epsilon_{\min }, \epsilon_{\max }\right]=[0.5,1.5] \mathrm{m}$.

27. Same as [25], except $\left[\epsilon_{\min }, \epsilon_{\max }\right]=[1,2] \mathrm{m}$.

28. Same as [25], except $\left[\epsilon_{\min }, \epsilon_{\max }\right]=[1.5,2.5] \mathrm{m}$.

29. Same as $[25]$, except $\left[\epsilon_{\min }, \epsilon_{\max }\right]=[2,2.5] \mathrm{m}$.

30. Same as $[25]$, except $\left[\epsilon_{\min }, \epsilon_{\max }\right]=[3,3.5] \mathrm{m}$.

31. Qualitative particle trajectory in the $(\dot{x}, \dot{y})$ phase plane for the Hamiltonian (2). In the oval regions, a resonant interaction occurs which may lead to a substantial phase change and thus to stochastic particle dynamics. This effect weakens as the gyroradius of a particular trajectory increases. 
Table I: Summary of Turbulent Spectrum Heating Simulations.

\begin{tabular}{|c|c|c|c|c|}
\hline Run & Wave Parameters & $\epsilon_{\min }-\epsilon_{\max }(\mathrm{m})$ & $\rho_{\max }(\mathrm{m})$ & $\tau_{\max }(\mathrm{eV})$ \\
\hline $1 \mathrm{~A}$ & $\multirow[t]{6}{*}{\nu_{i}^{(A)}, k_{i}^{(A)}, \varphi_{i}^{(A)}}_{i=1}^{10}$ & $0-1$ & 14 & $1.5 \times 10^{1}$ \\
\hline $2 A$ & & $0.5-1.5$ & 29 & $6.4 \times 10^{1}$ \\
\hline $3 A$ & & $1-2$ & 51 & $2.0 \times 10^{2}$ \\
\hline $4 A$ & & $1.5-2.5$ & 80 & $4.9 \times 10^{2}$ \\
\hline $5 A$ & & $2-2.5$ & 83 & $5.3 \times 10^{2}$ \\
\hline $6 A$ & & $3-3.5$ & 133 & $1.4 \times 10^{3}$ \\
\hline 1B & $\multirow{6}{*}{\nu_{i}^{(B)}, k_{i}^{(B)}, \varphi_{i}^{(B)}}_{i=1}^{10}$ & $0-1$ & 4 & $1.2 \times 10^{0}$ \\
\hline 2B & & $0.5-1.5$ & 5 & $1.9 \times 10^{\circ}$ \\
\hline 3B & & $1-2$ & 6 & $2.8 \times 10^{0}$ \\
\hline $4 B$ & & $1.5-2.5$ & 60 & $2.8 \times 10^{2}$ \\
\hline $5 B$ & & $2-2.5$ & 84 & $5.4 \times 10^{2}$ \\
\hline $6 \mathrm{~B}$ & & $3-3.5$ & 108 & $8.9 \times 10^{2}$ \\
\hline
\end{tabular}


Surface of Section

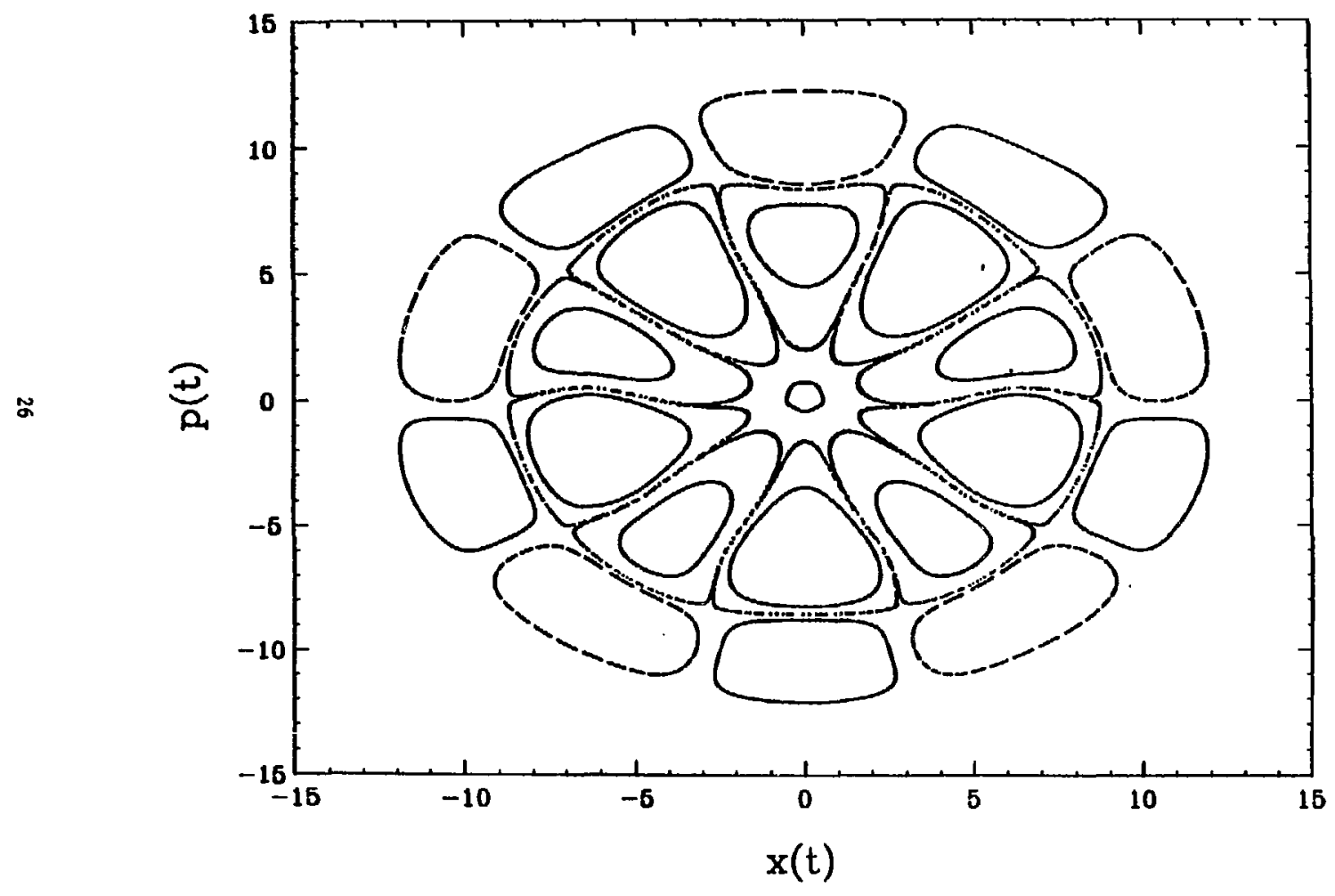

Flg. 1 
Surface of Section

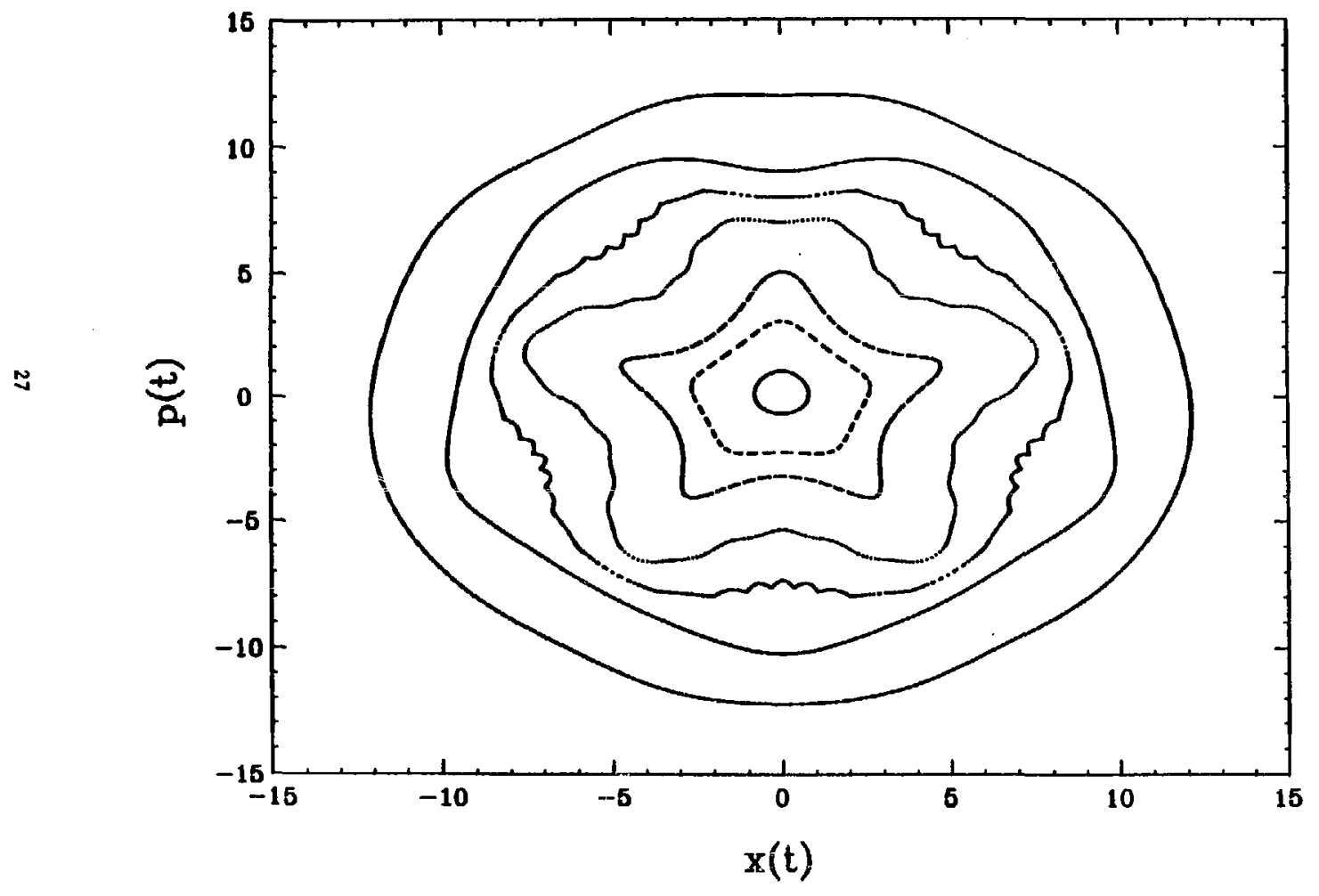

F1 . 2 
Surface of Section

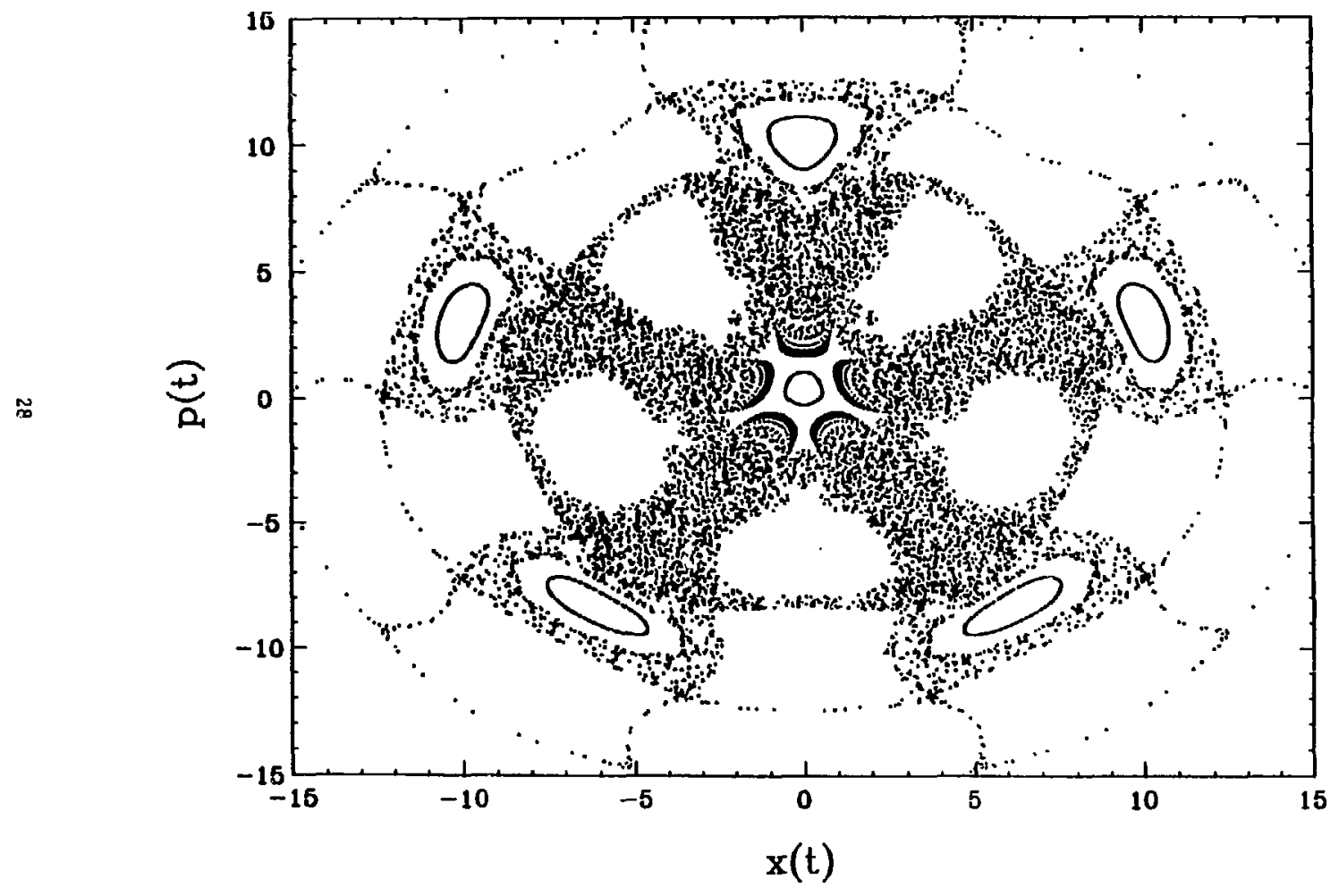




\section{Surface of Section}

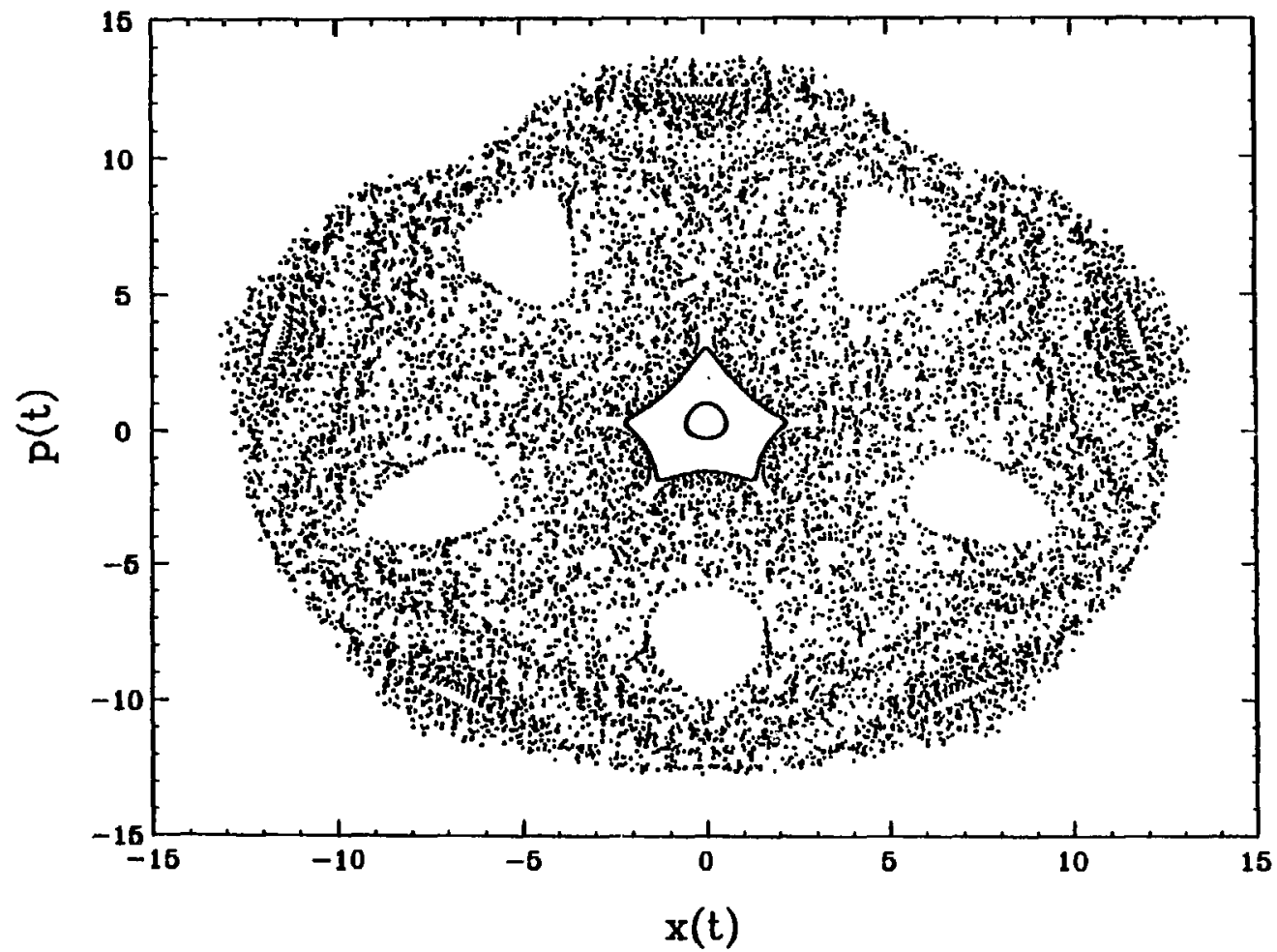

Fig - 4 


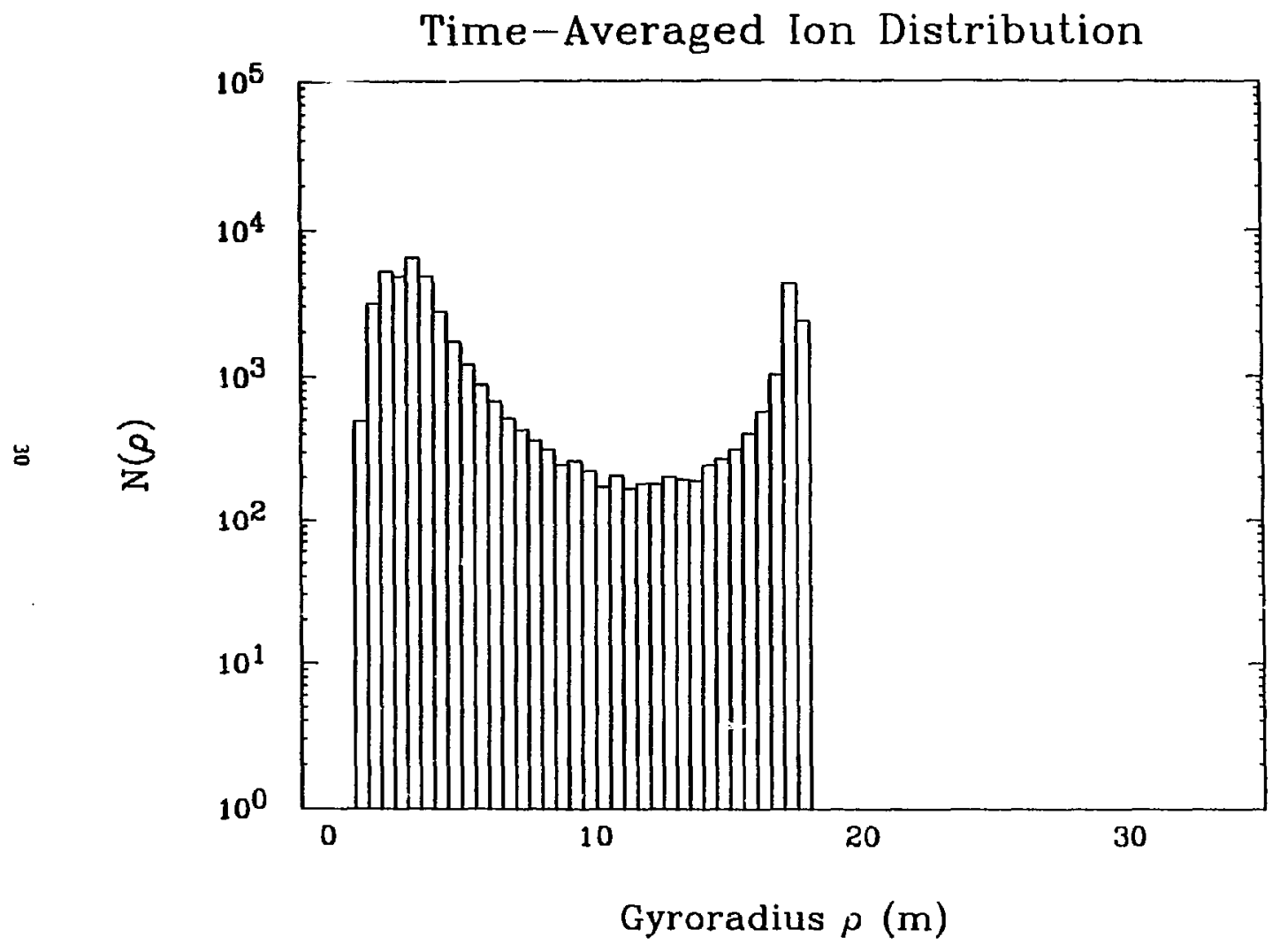

Fig. 5 
Time-Averaged Ion Distribution

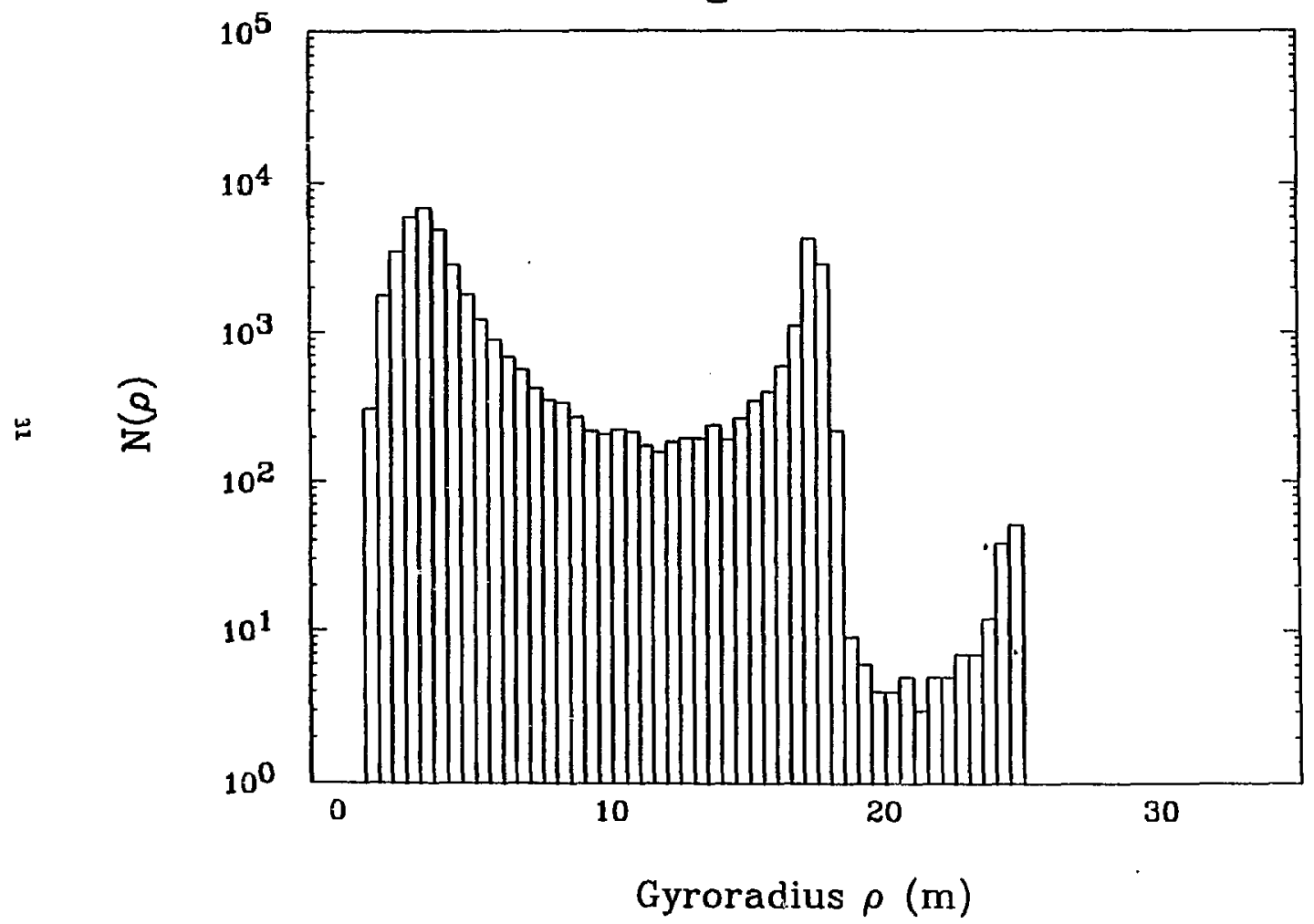

B18. 6 
Time-Averaged Ion Distributior

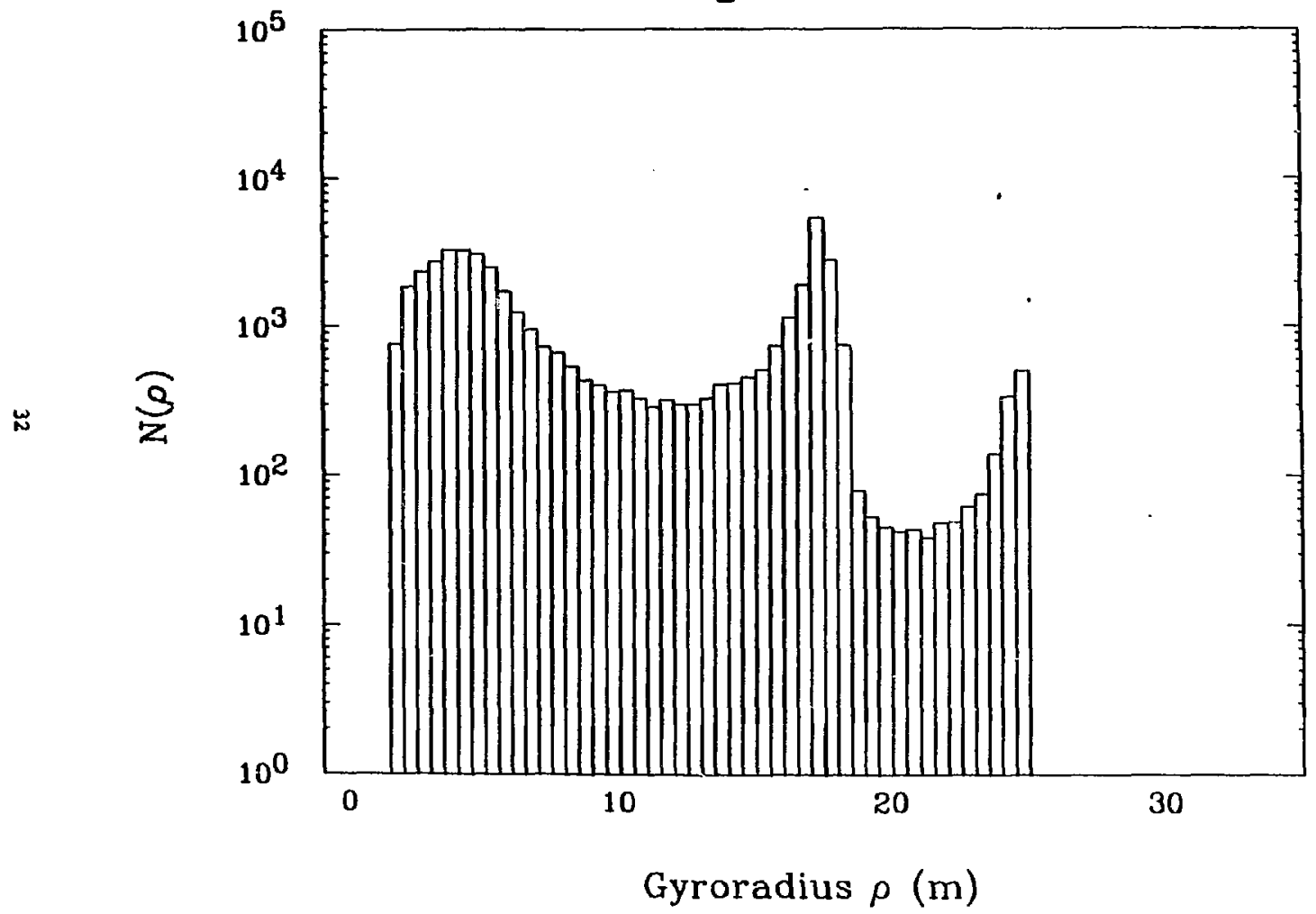

F1g. 7 
Time-Averaged Ion Distribution

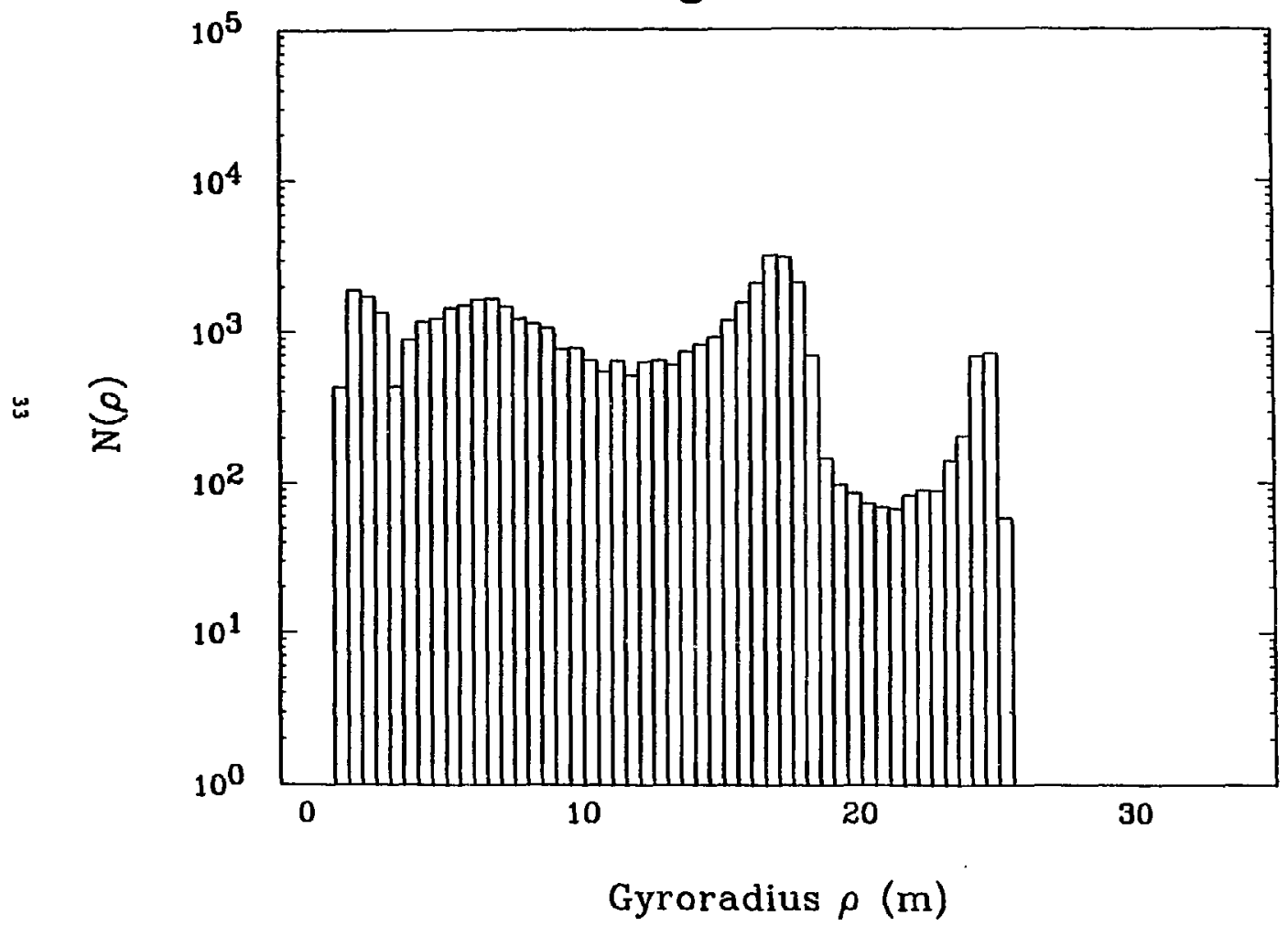

Fig. 8 
Time-Averaged Ion Distribution

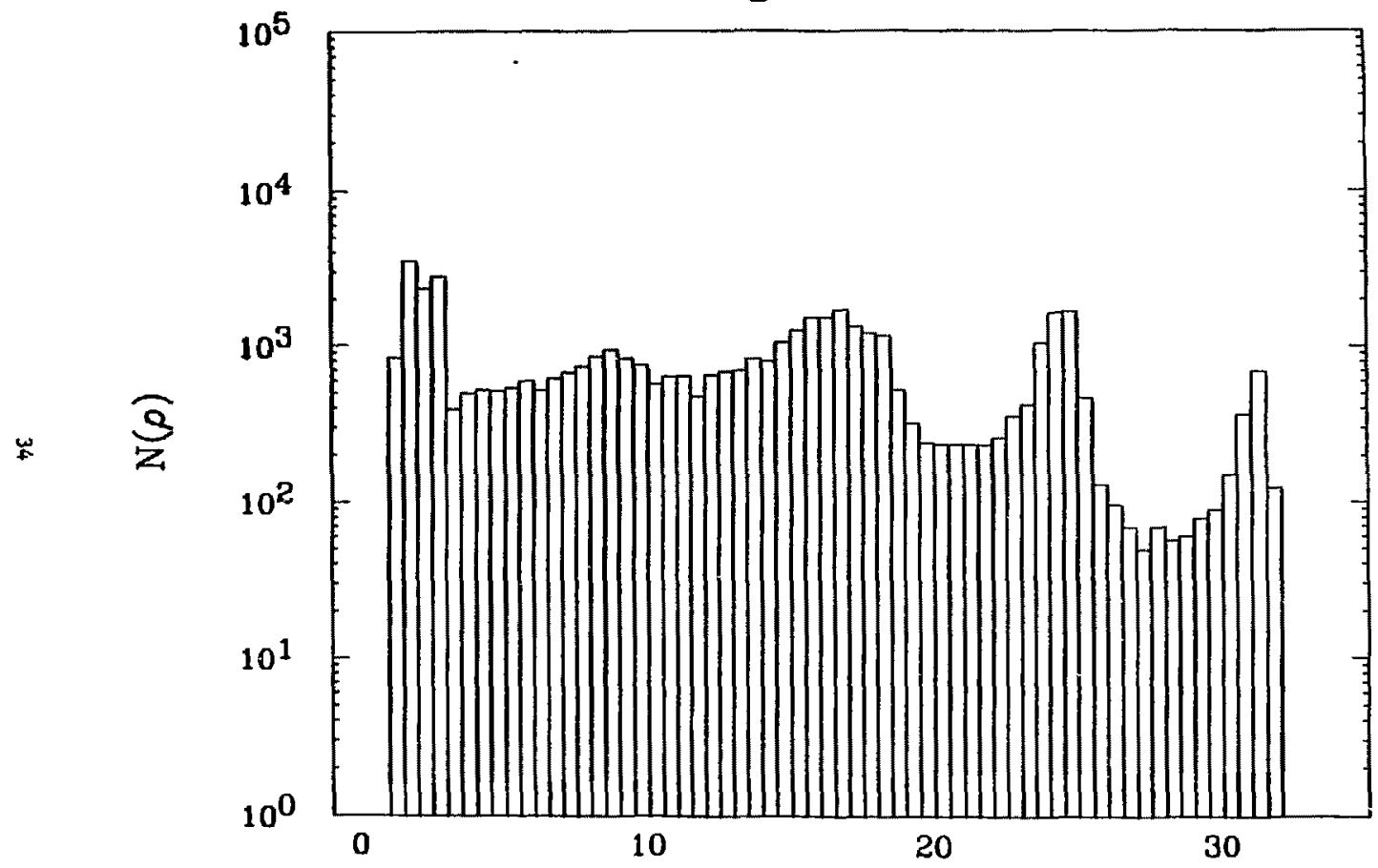

Gyroradius $\rho(\mathrm{m})$ 
Time-Averaged Ion Distribution

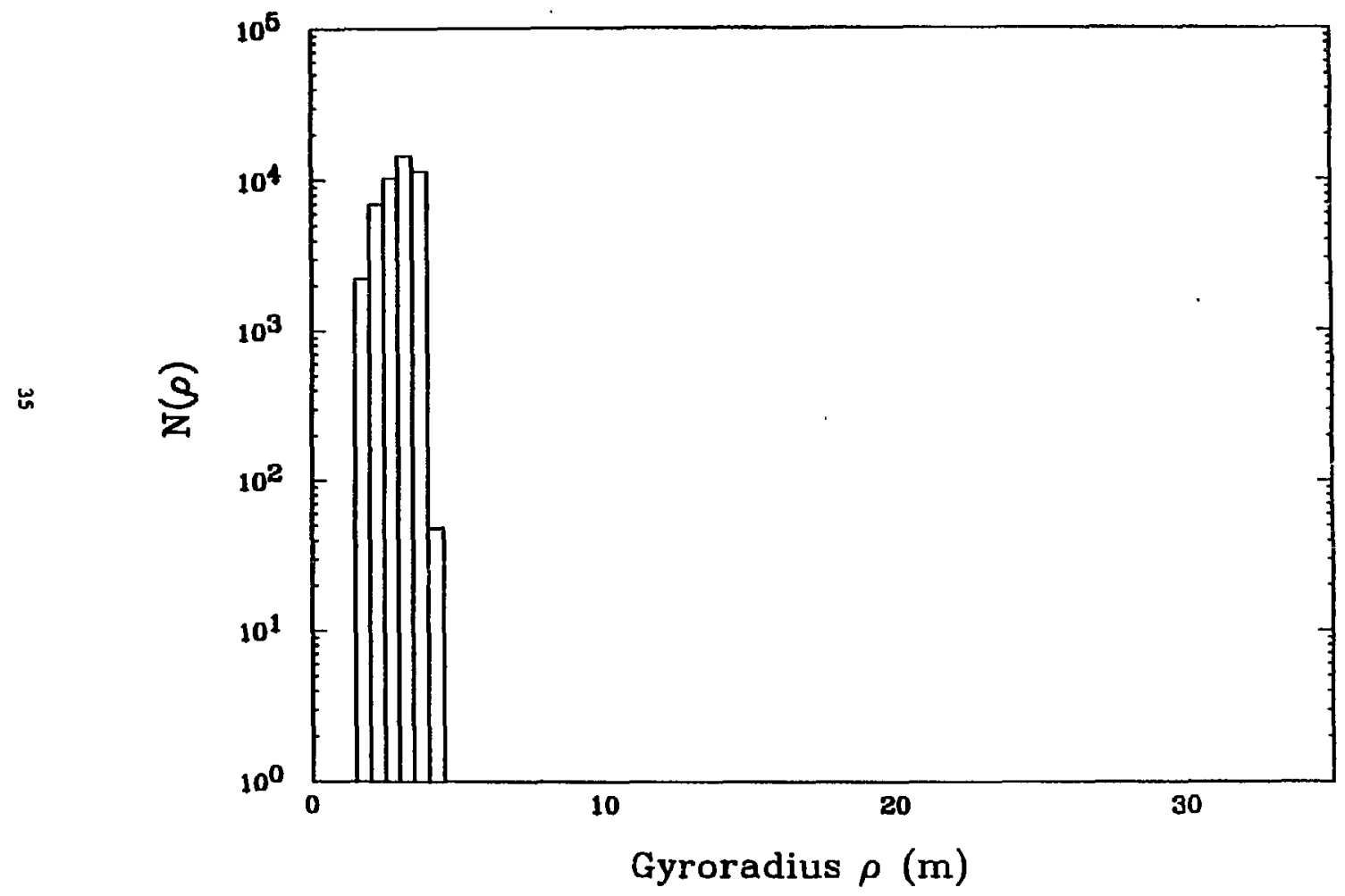

F18. 10 
Time-Averaged Ion Distribution

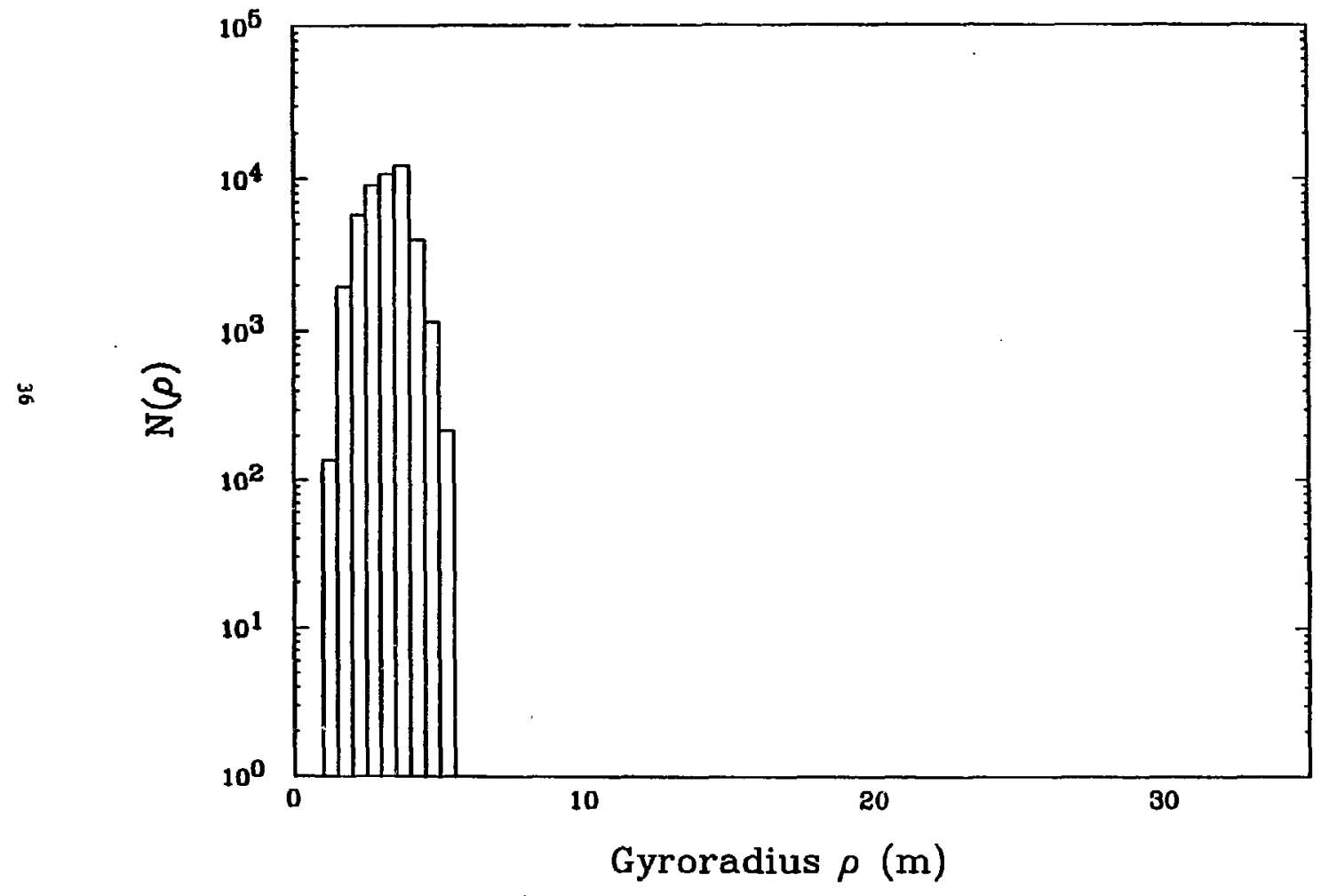

F18. 11 
Time-Averaged Ion Distribution

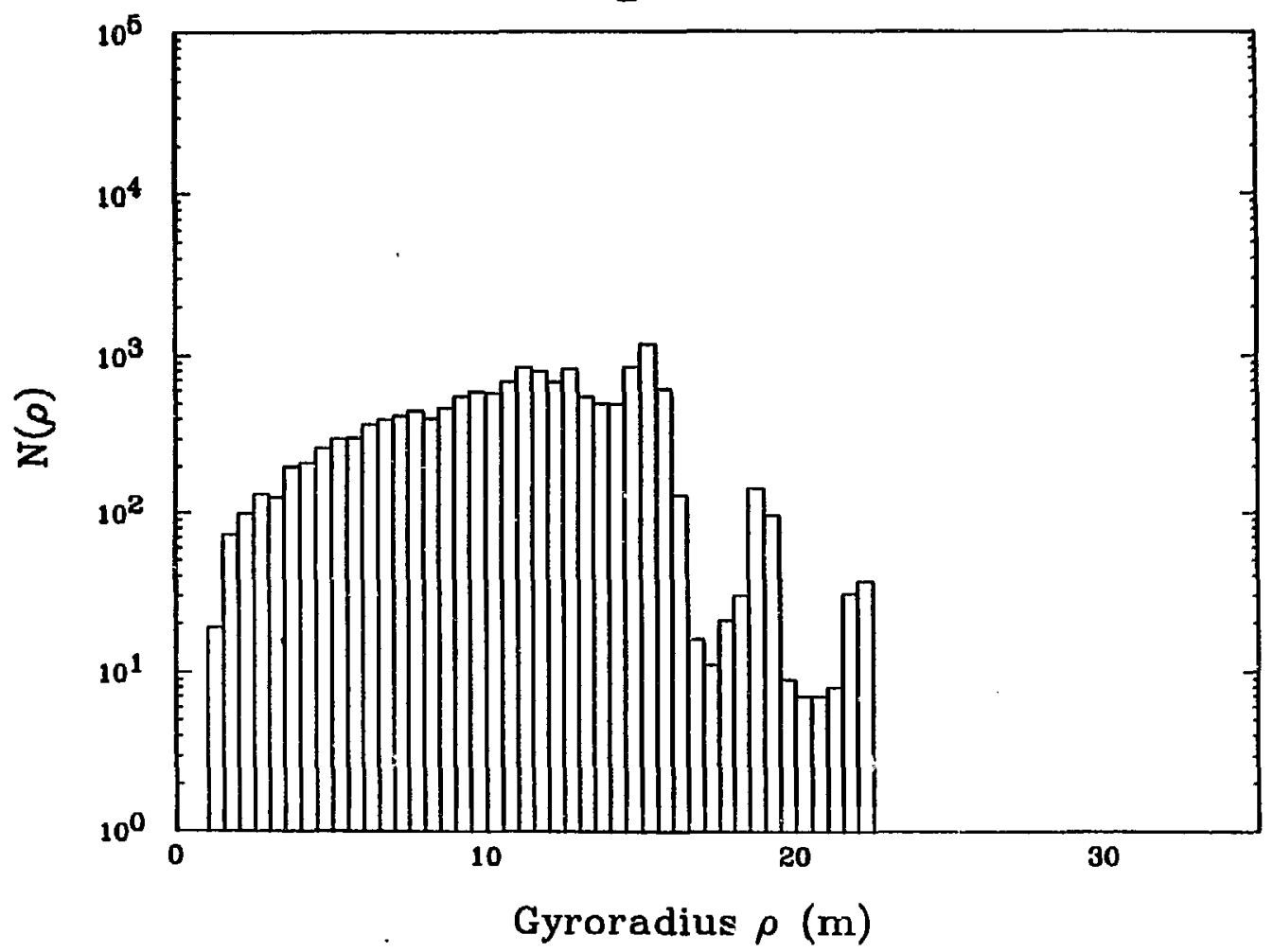

Ff. 12 
Time-Averaged Ion Distribution

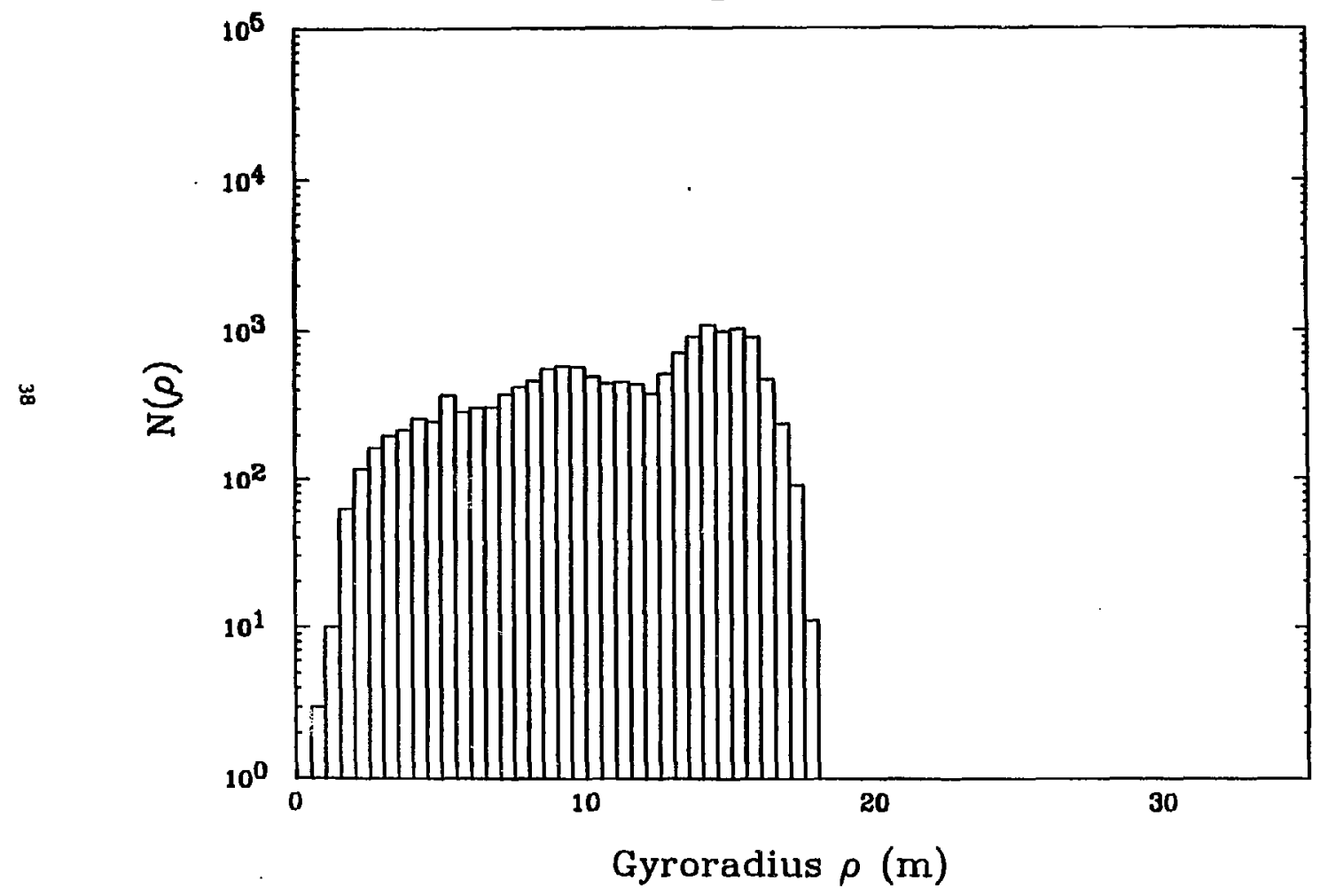




\section{Electric Field Amplitude}

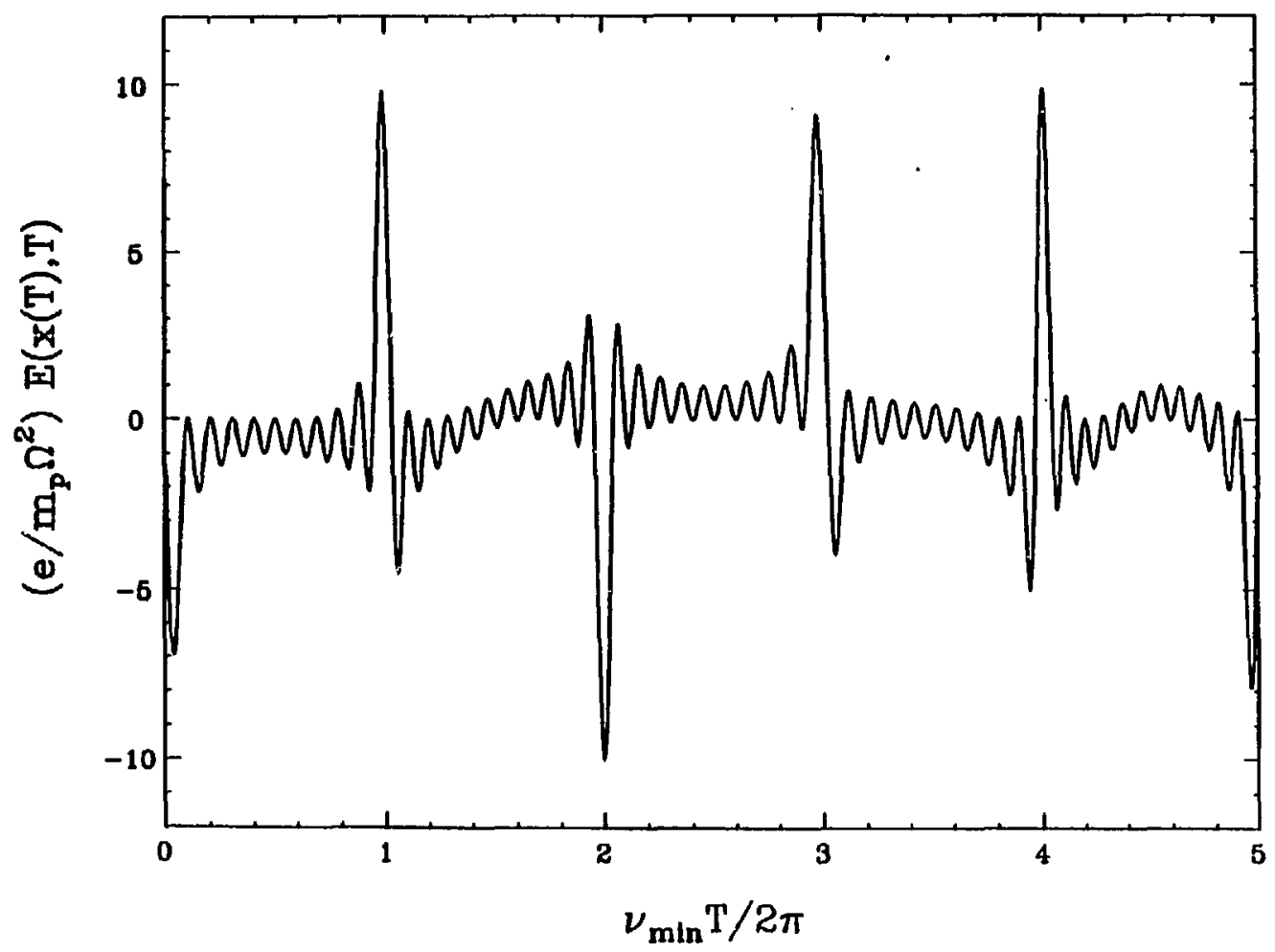

Fig. 14 
Electric Field Amplitude

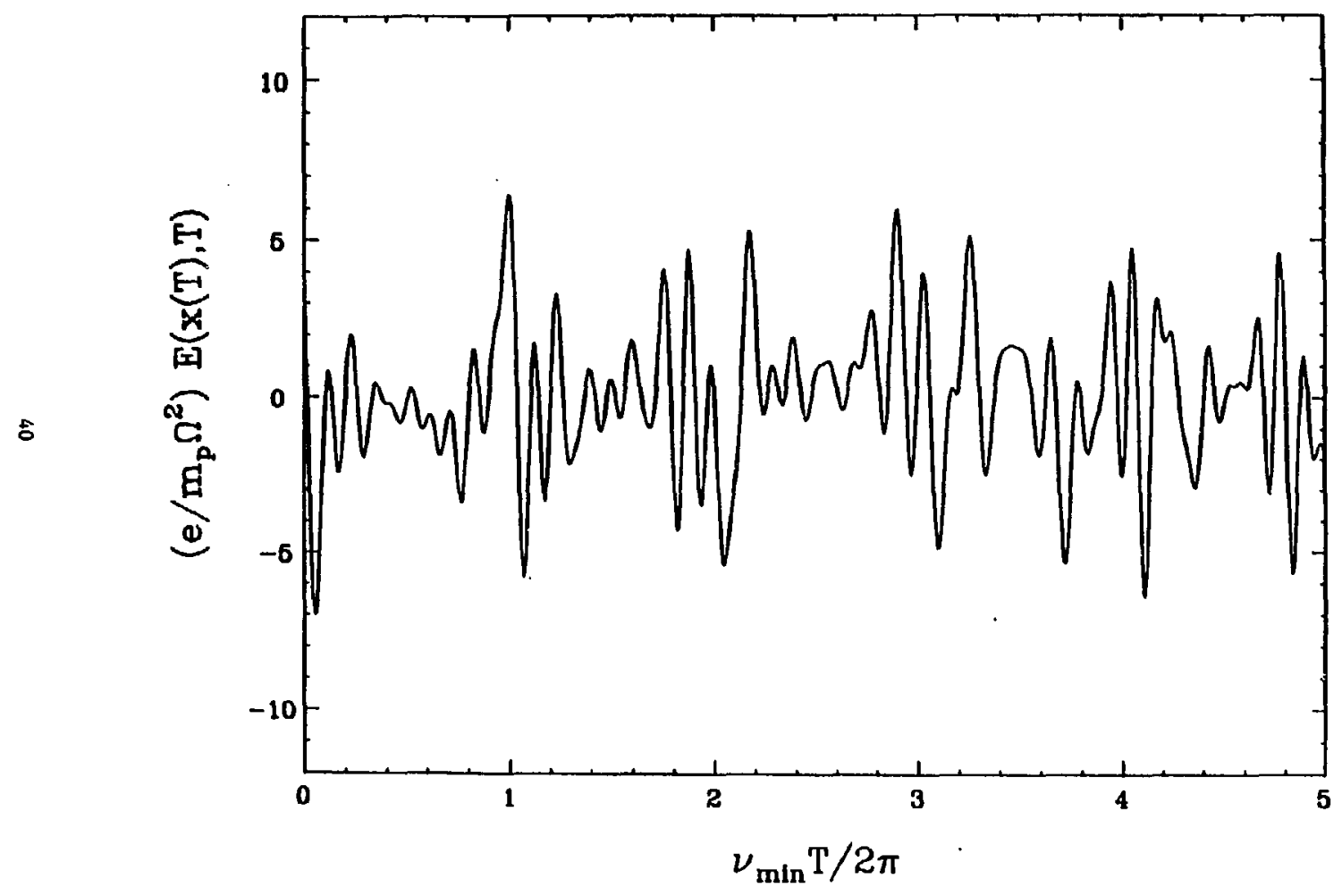

F1g. 15 
Surface of Section

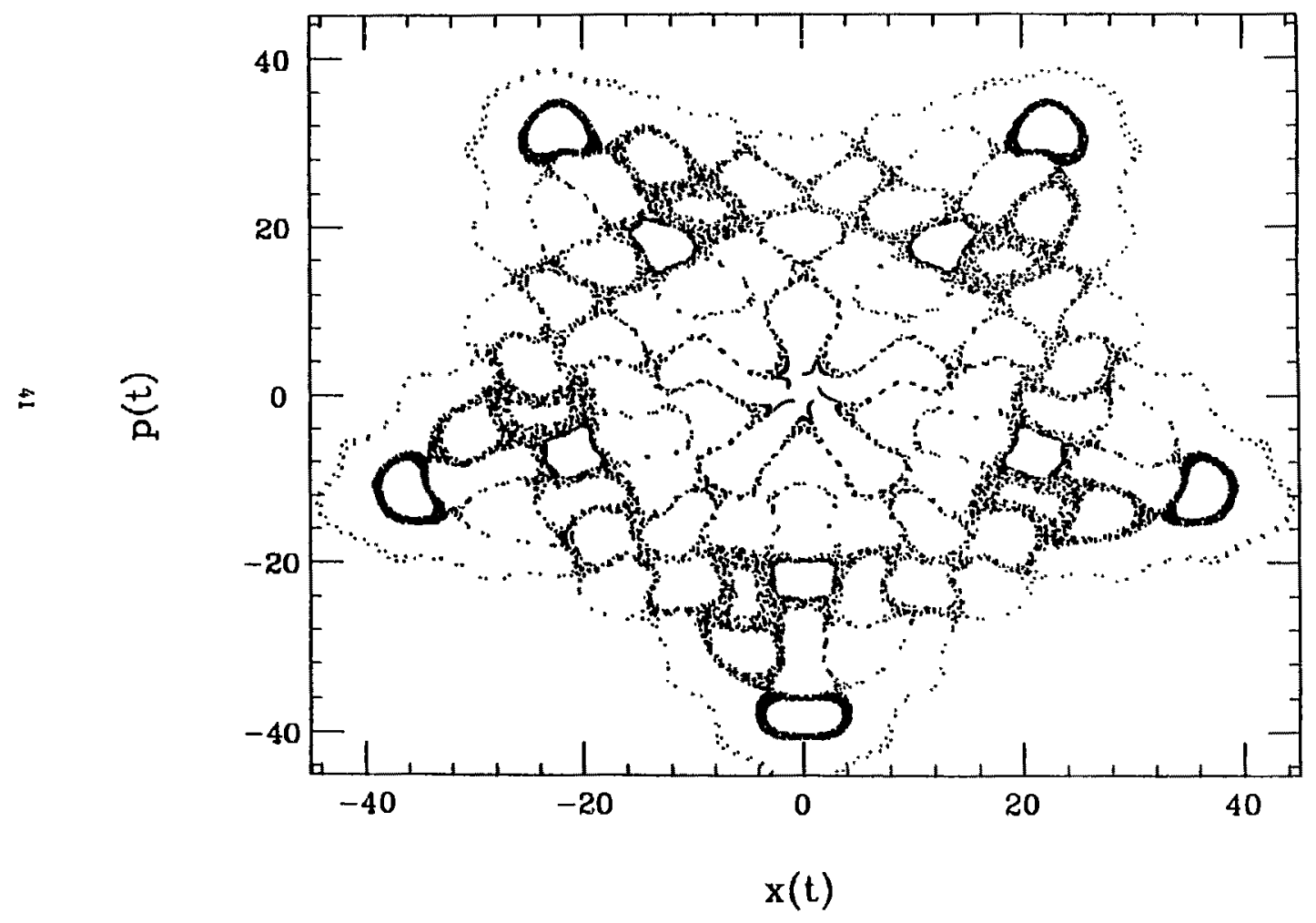

F1g. 16 
Surface of Section

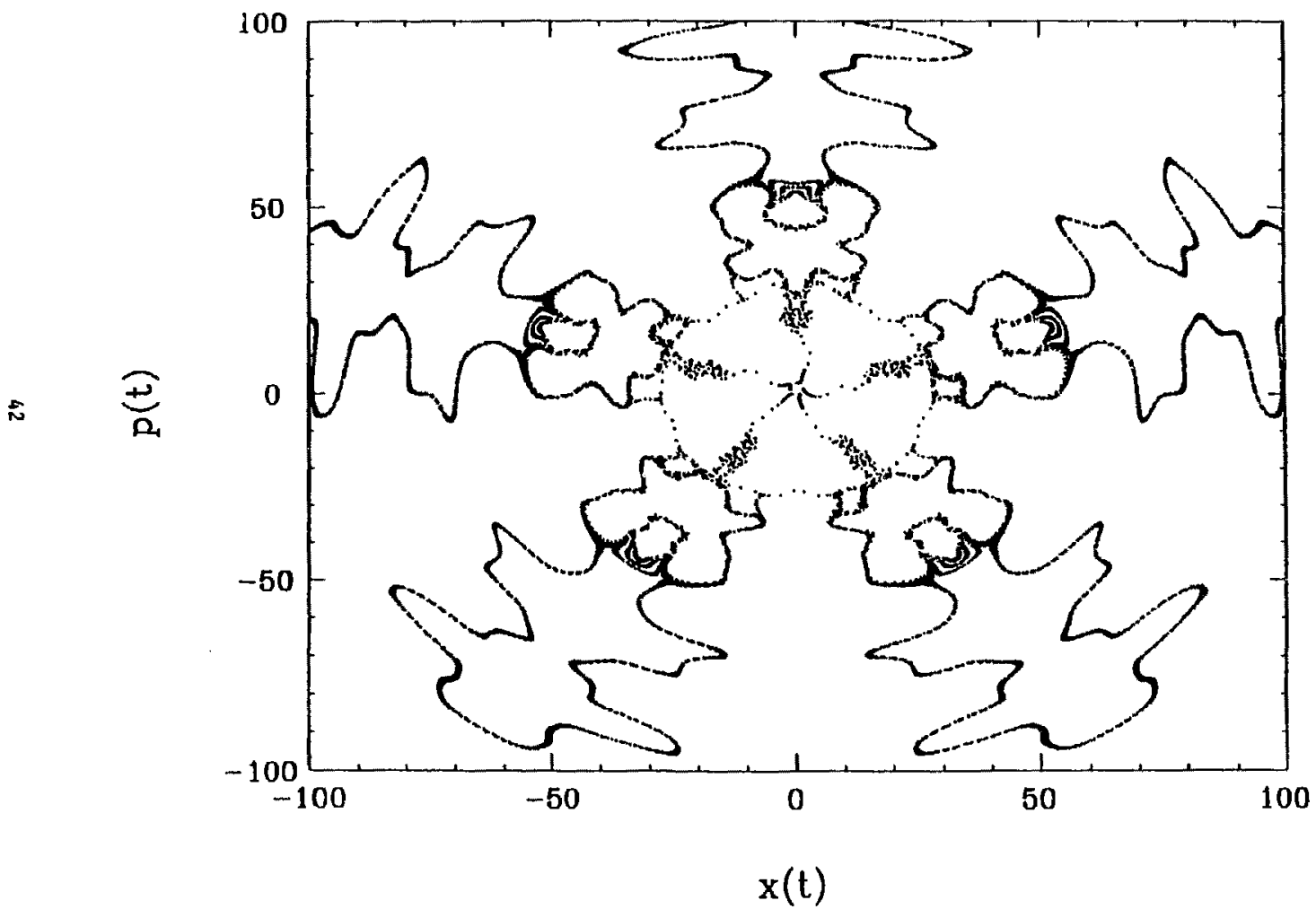

F1g. 17 


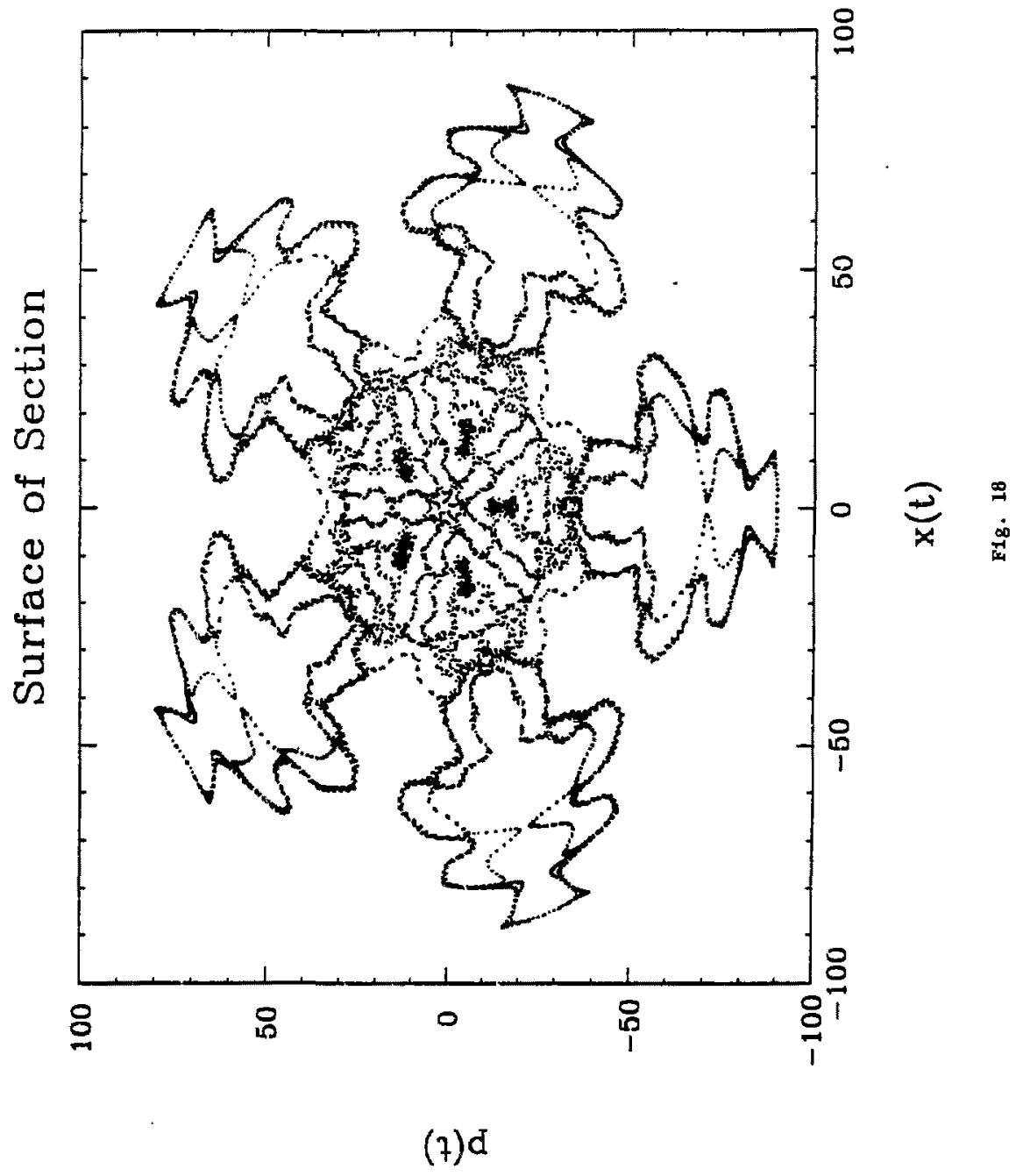


Time-Averaged Ion Distribution

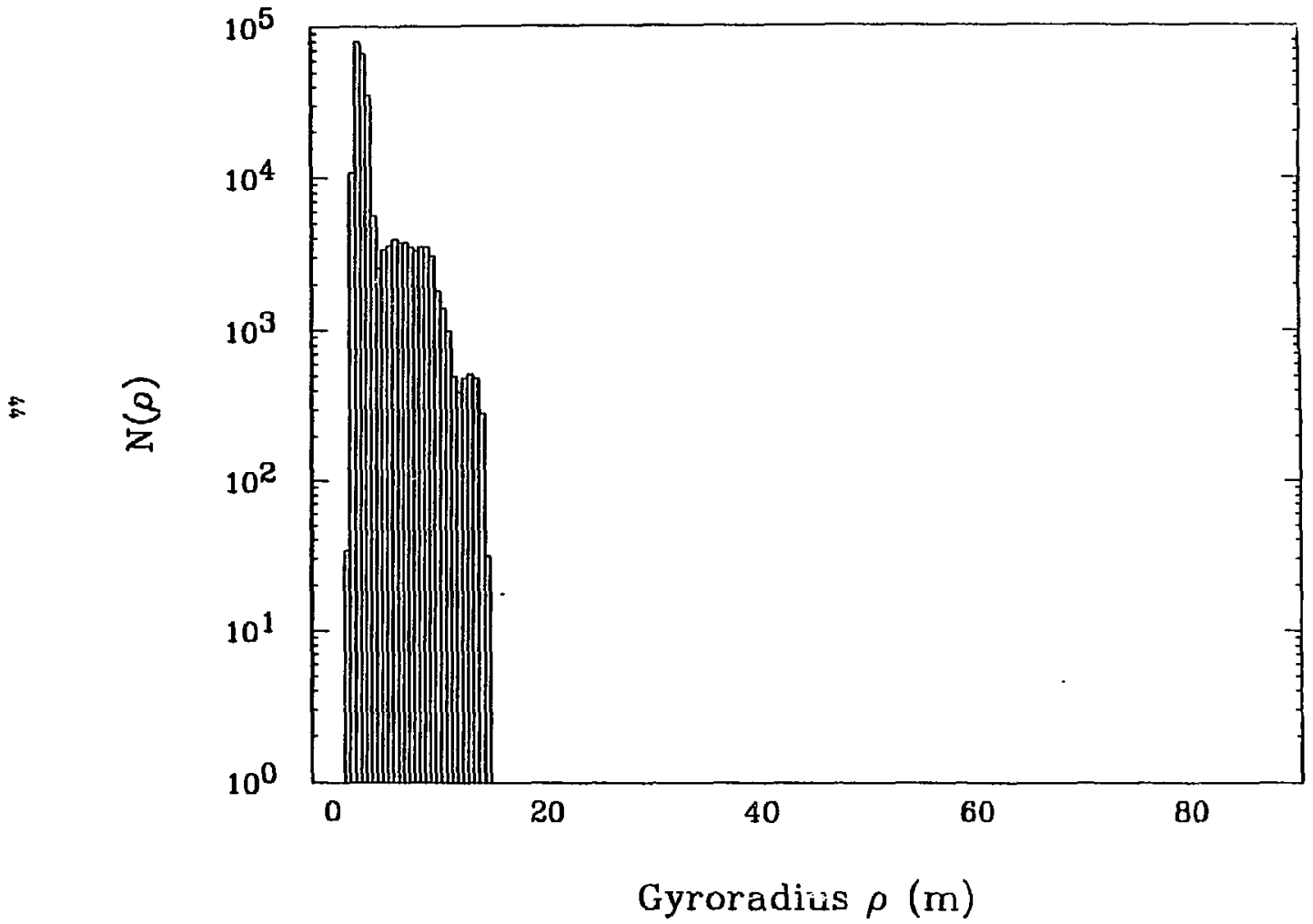

F1g. 19 


\section{Time-Averaged Ion Distribution}

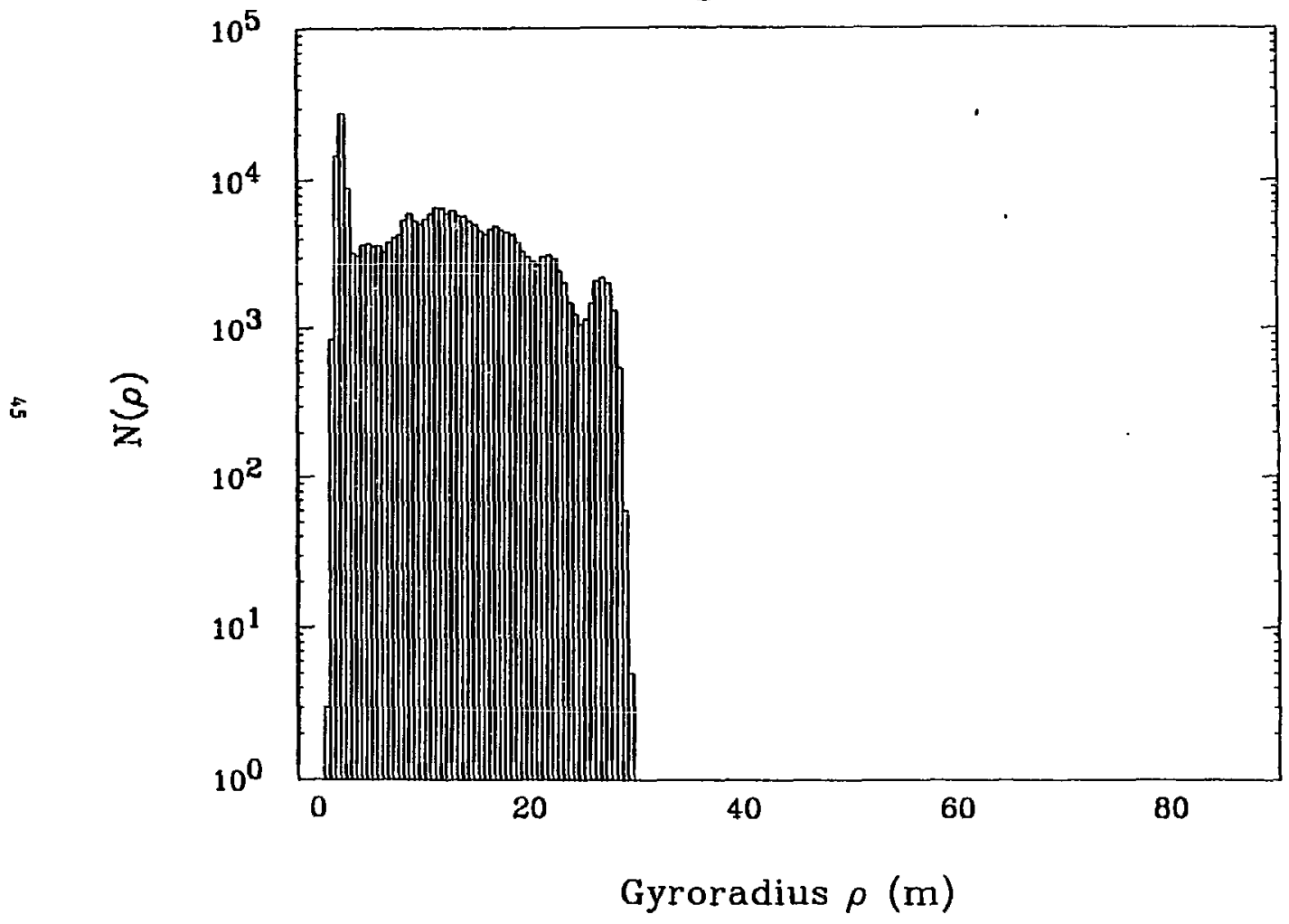

F1B. 20 
Time-Averaged Ion Distribution

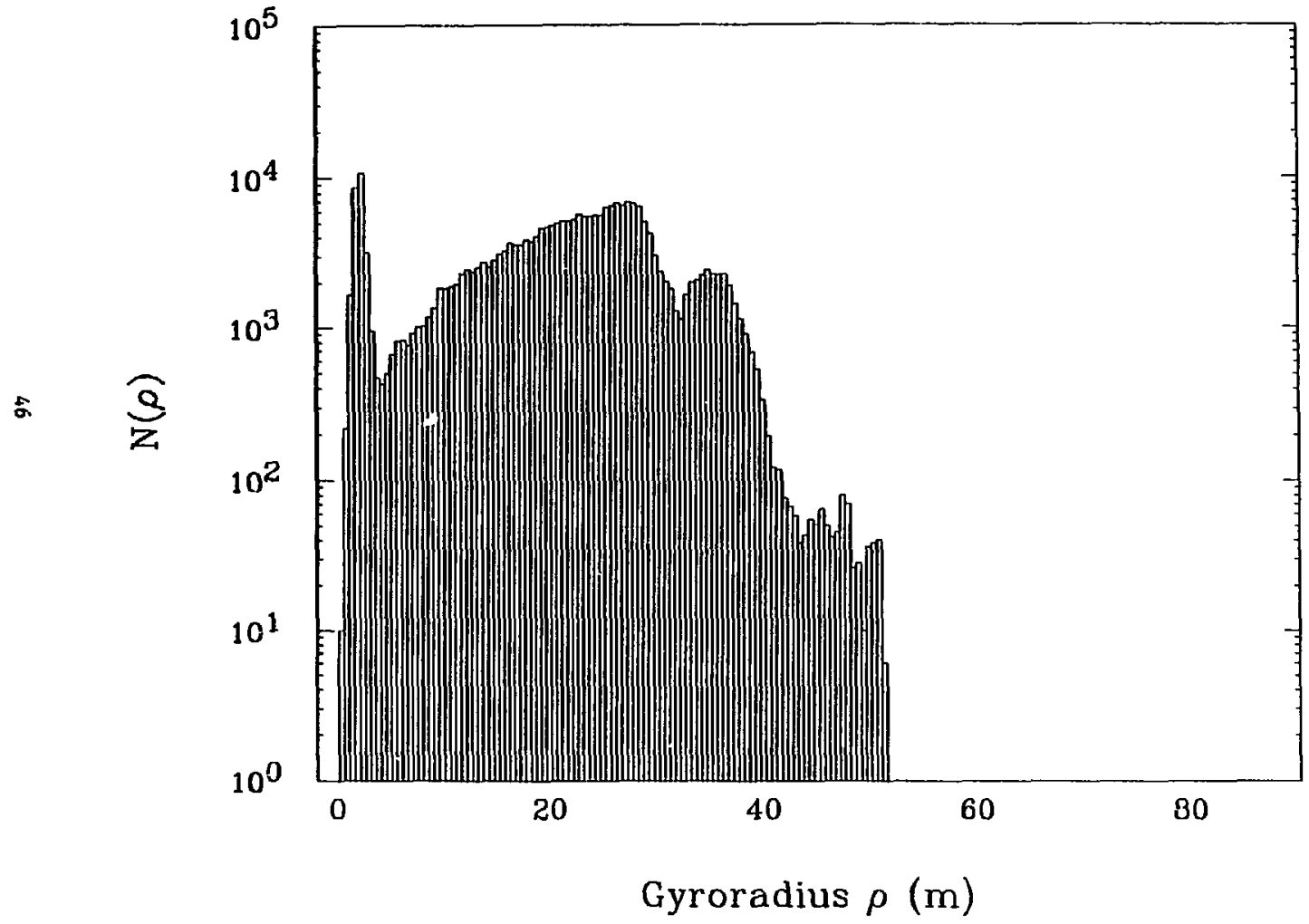

F18. 21 
Time-Averaged Ion Distribution

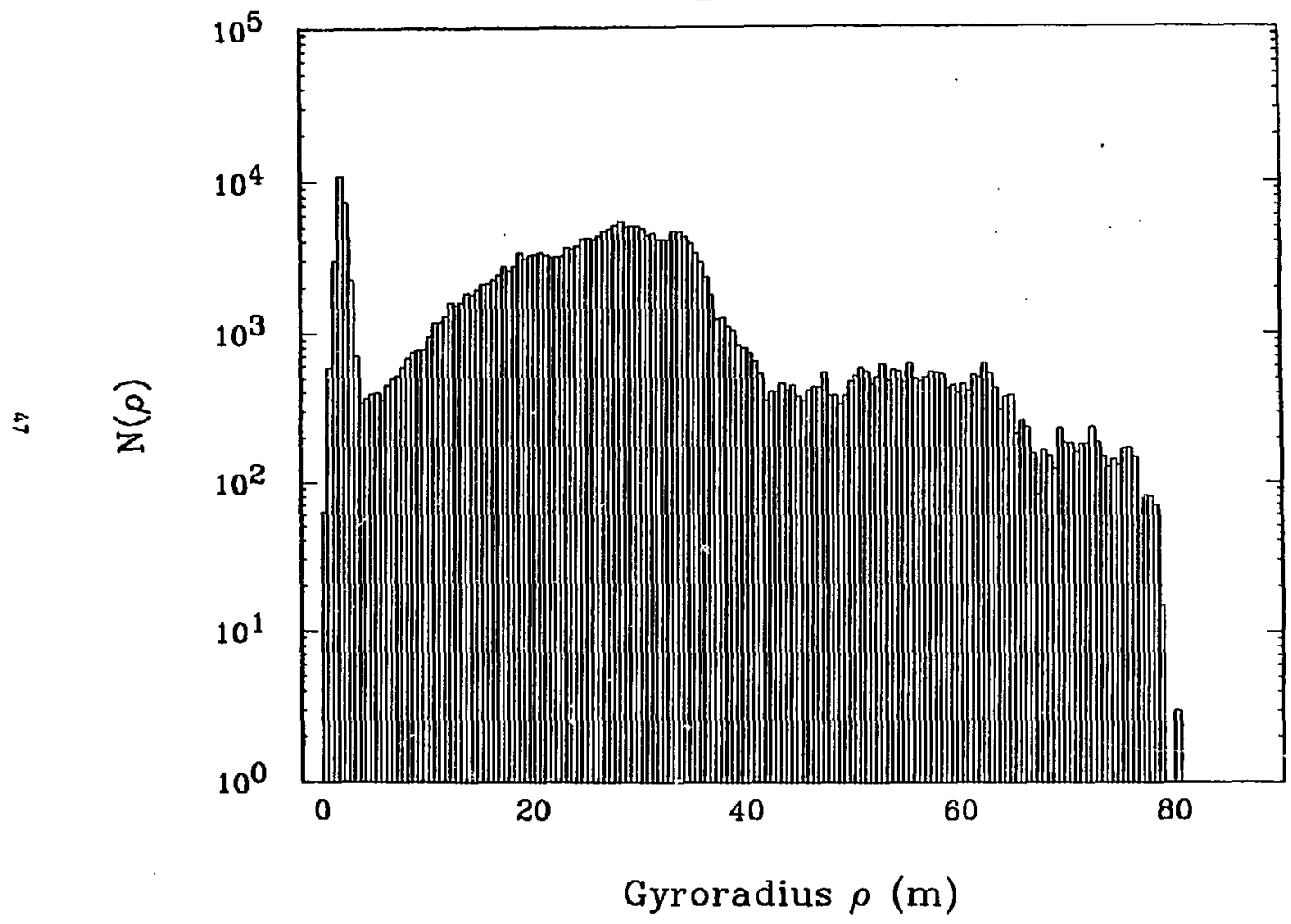

F1g. 22 
Time-Averaged Ion Distribution

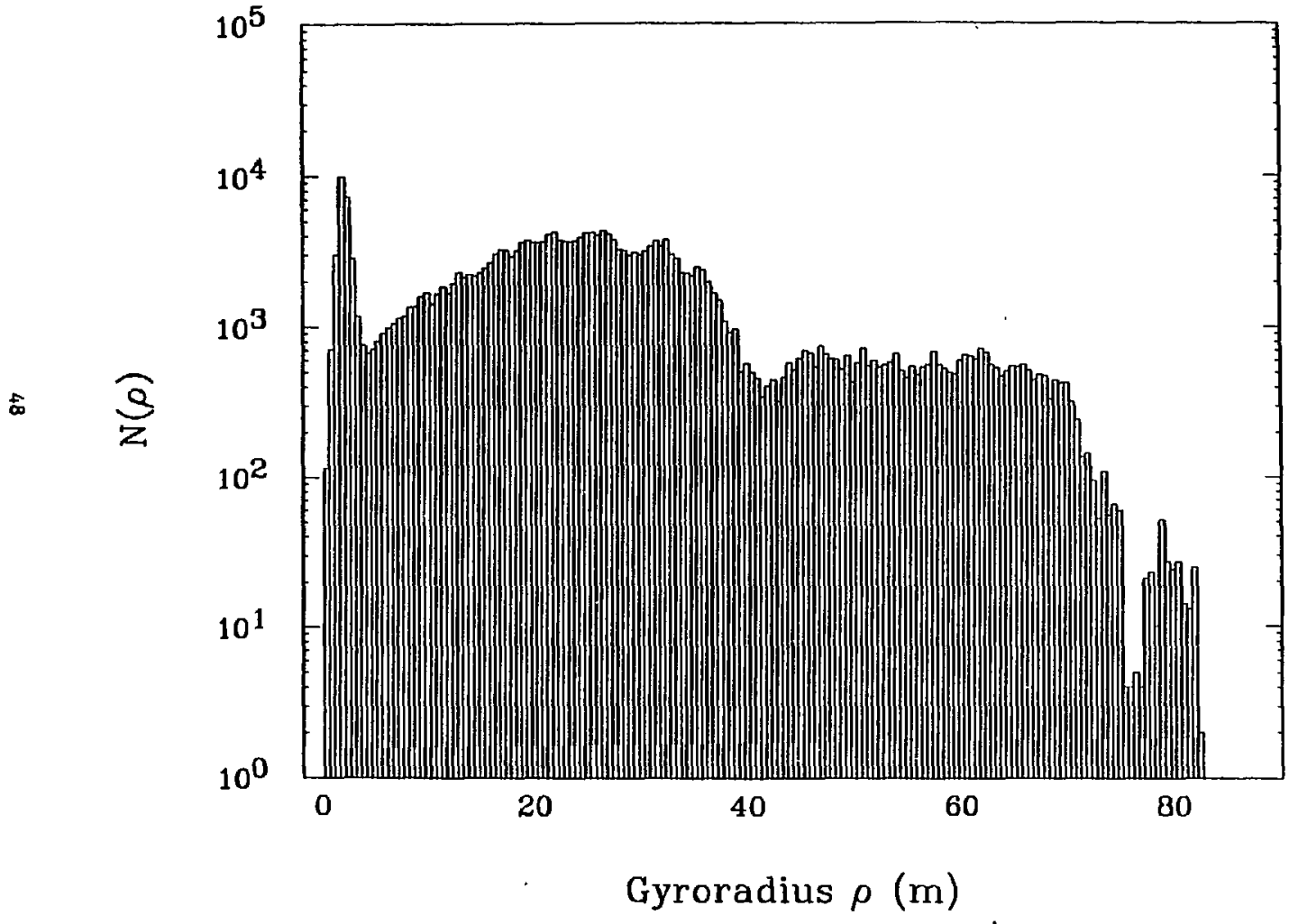

F1g. 23 
Time-Averaged Ion Distribution

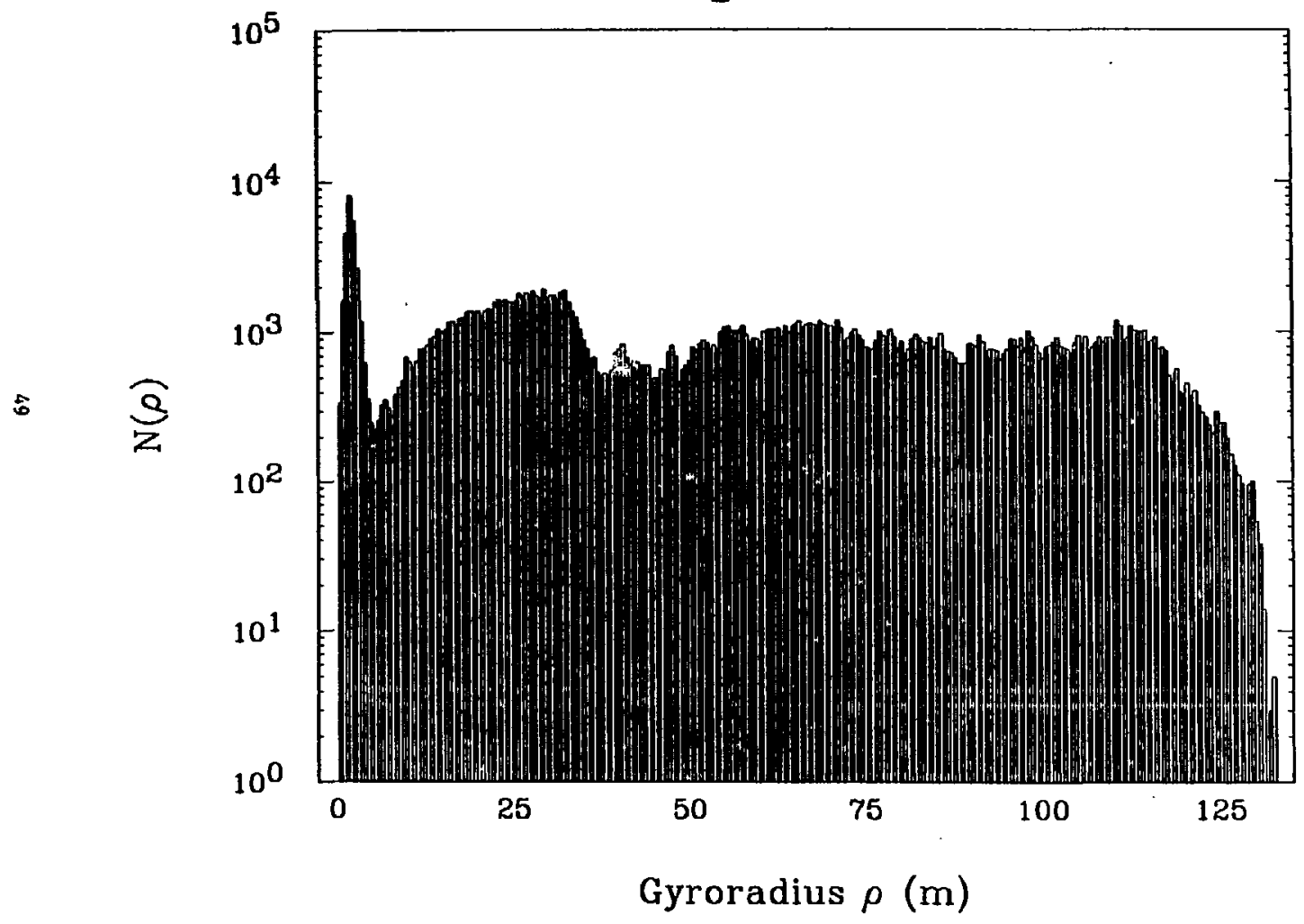

F1g. 24 
Time-Averaged Ion Distribution

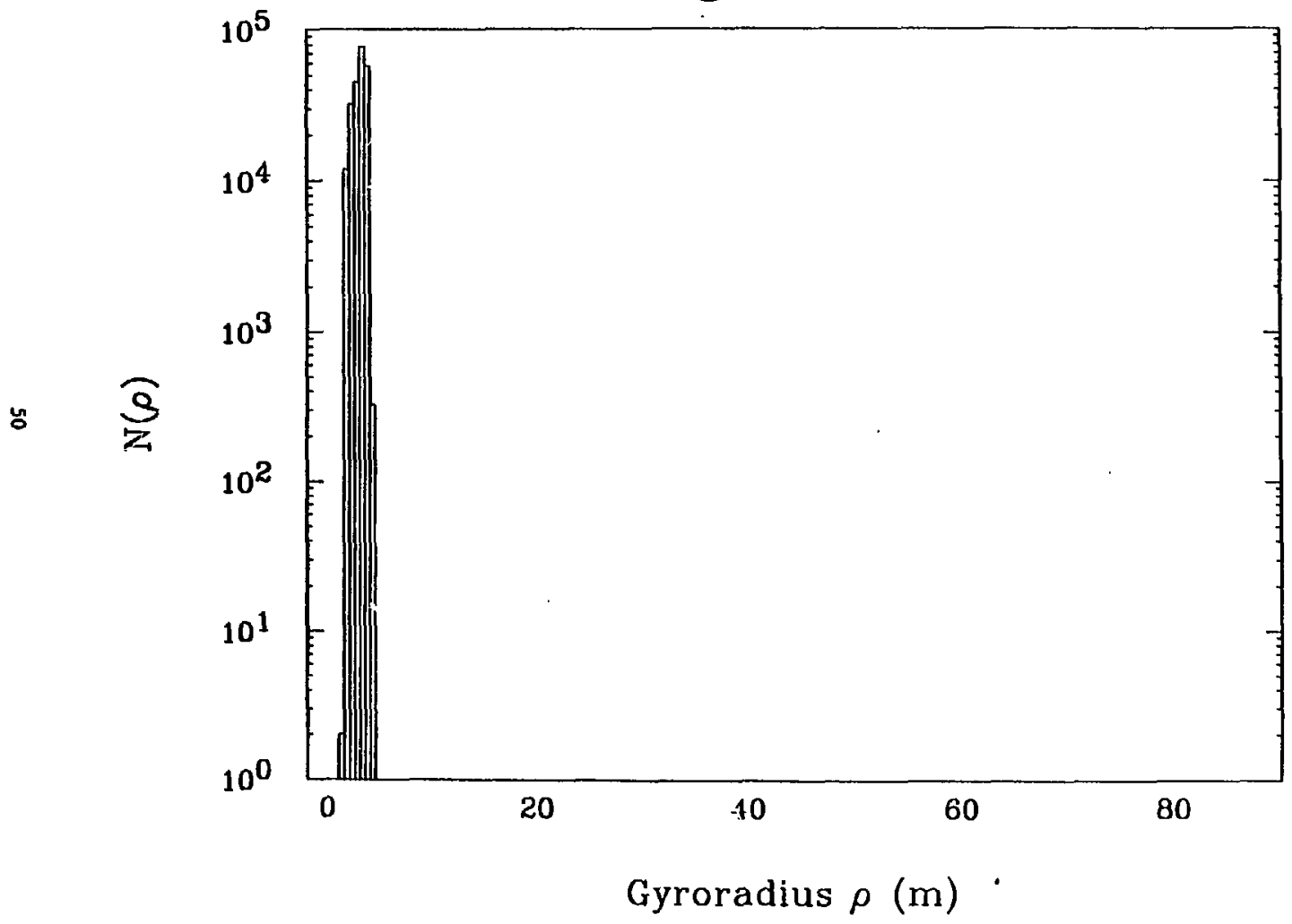

Ftg. 25 
Time-Averaged Ion Distribution

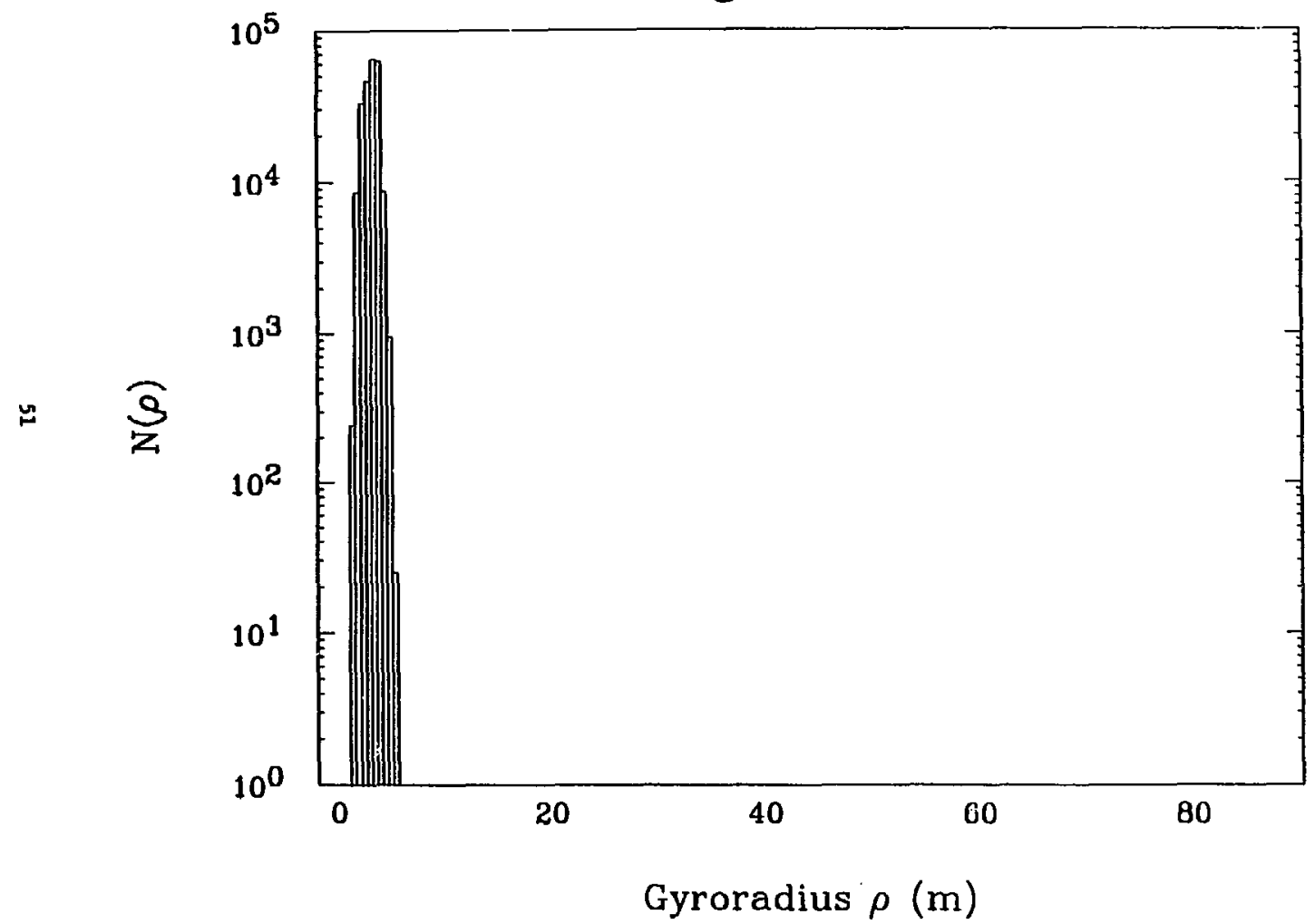

F18. 25 
Time-Averaged Ion Distribution

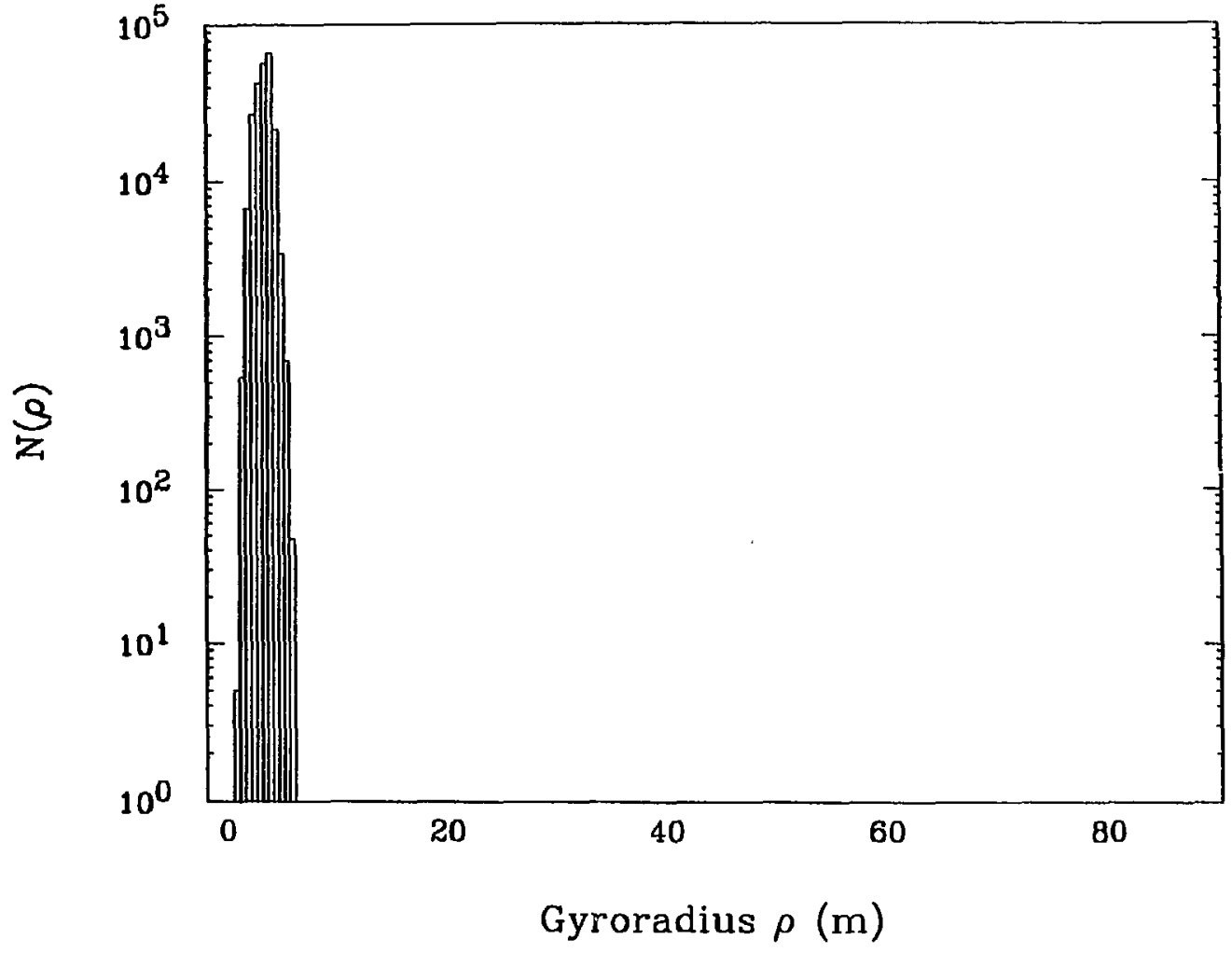

Ffg. 27 
Time-Averaged Ion Distribution

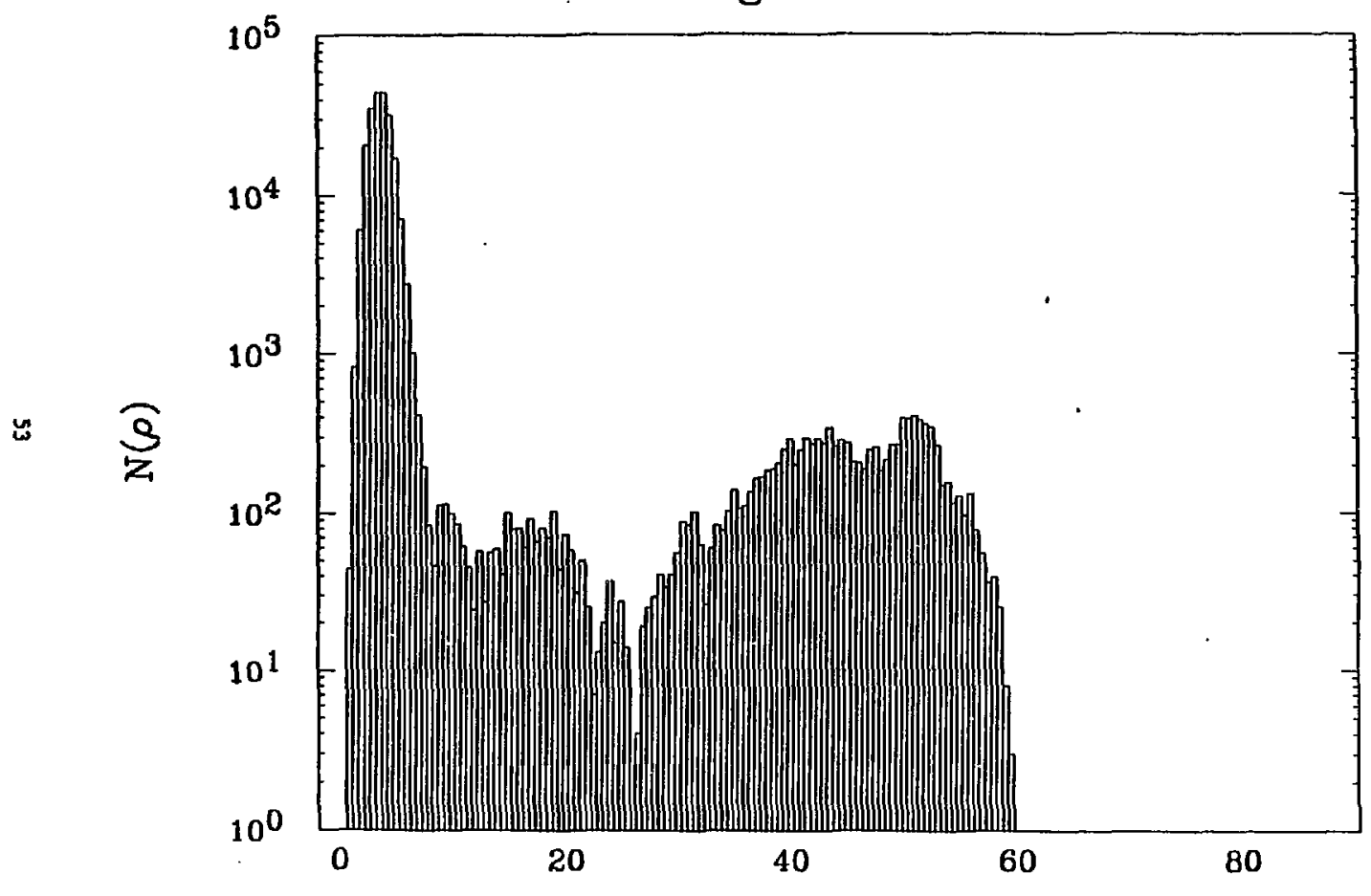

Gyroradius $\rho(\mathrm{m})$

F1g. 28 


\section{Time-Averaged Ion Distribution}

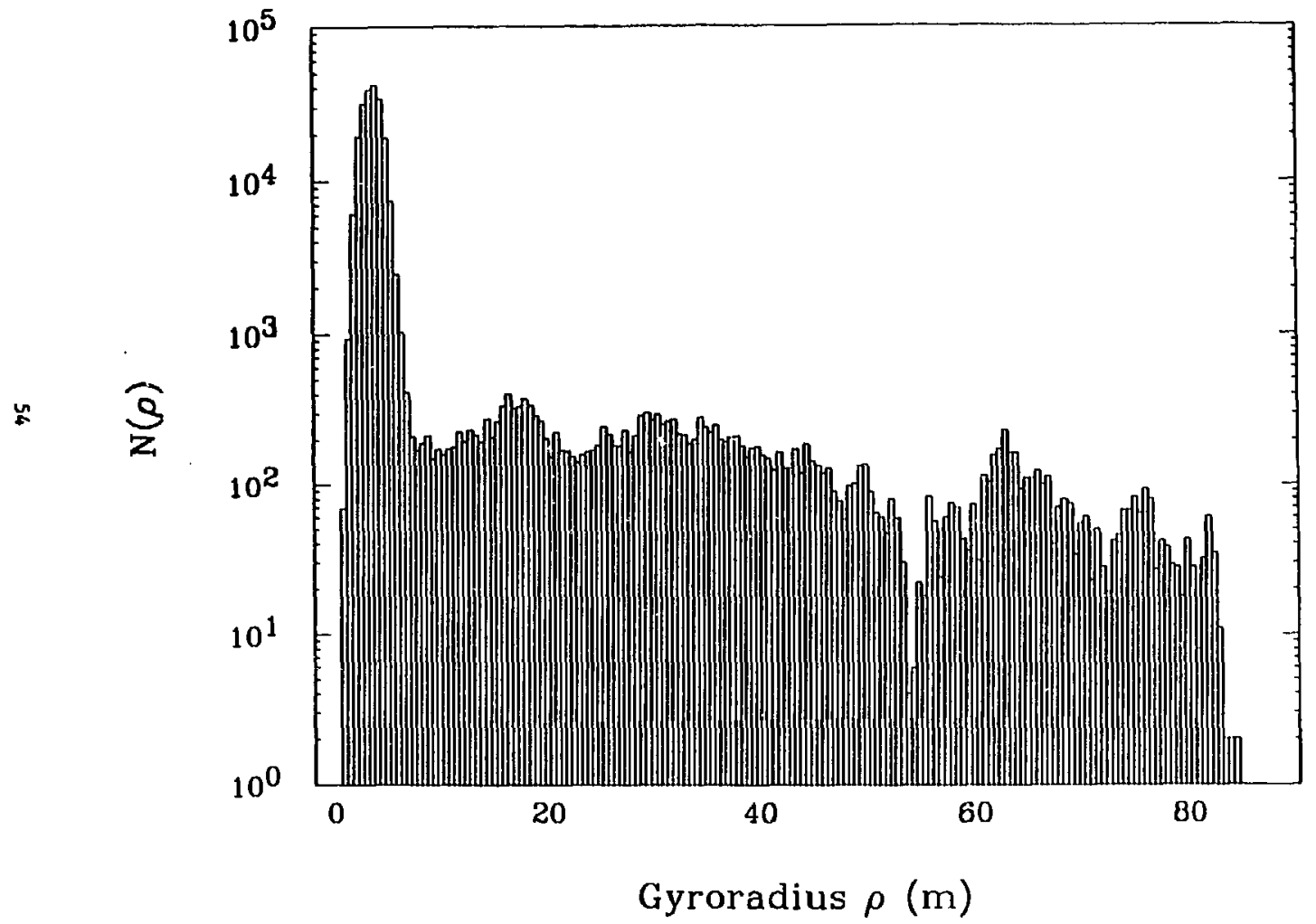


Time-Averaged Ion Distribution

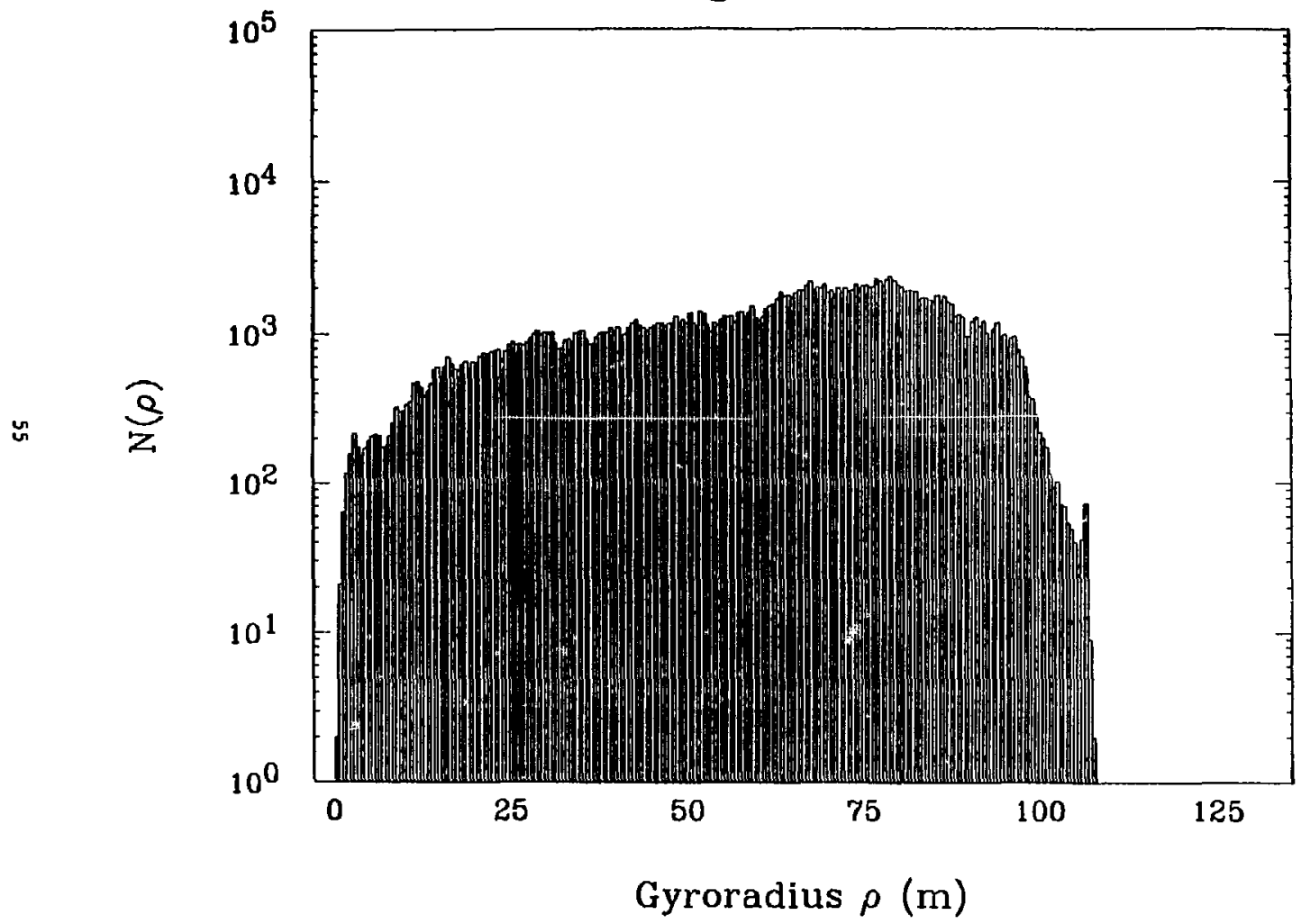

F18. 30 


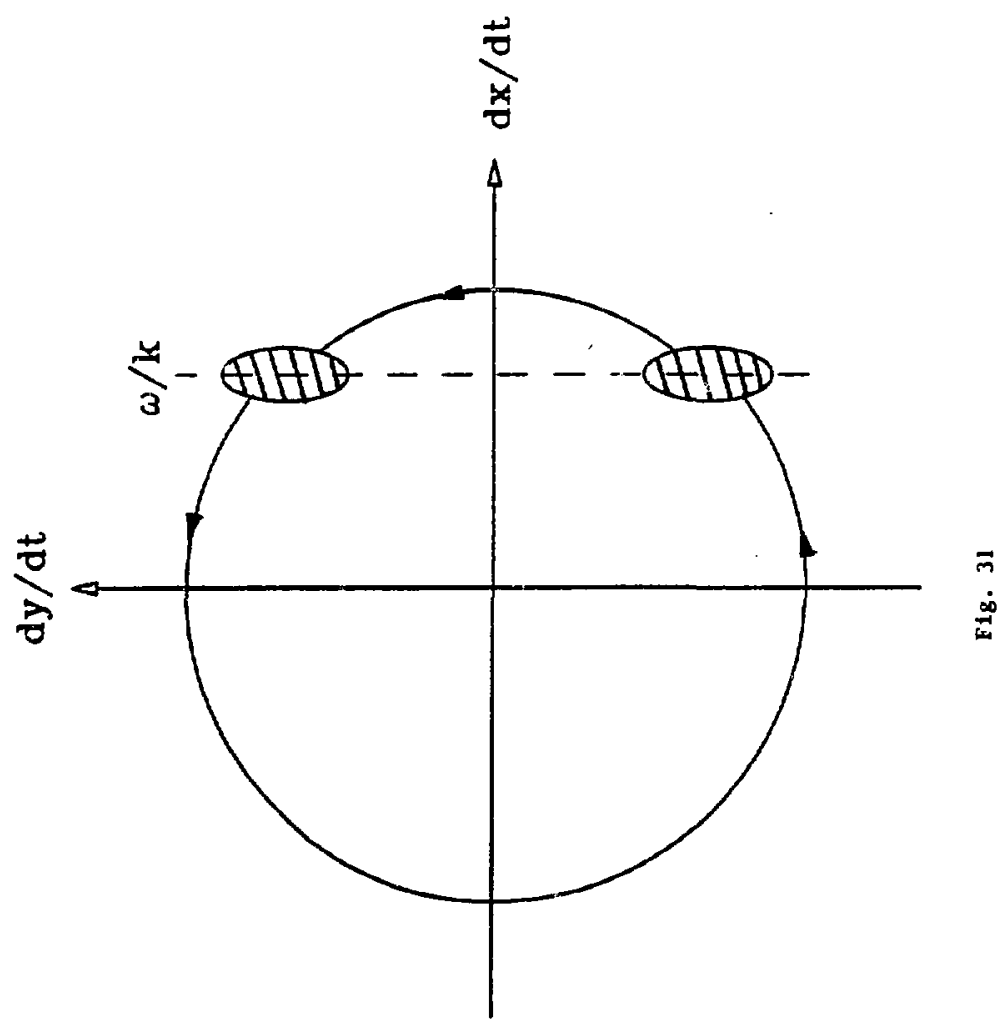

56 